\title{
Role of the plasmapause in dictating the ground accessibility of ELF/VLF chorus
}

\author{
D. I. Golden, ${ }^{1}$ M. Spasojevic, ${ }^{1}$ F. R. Foust, ${ }^{1}$ N. G. Lehtinen, ${ }^{1}$ N. P. Meredith, ${ }^{2}$ \\ and U. S. Inan ${ }^{1,3}$ \\ Received 21 July 2010; revised 23 August 2010; accepted 2 September 2010; published 17 November 2010
}

[1] This study explores the manner in which the plasmapause is responsible for dictating which magnetospheric source regions of ELF/VLF chorus are able to propagate to and be received by midlatitude stations on the ground. First, we explore the effects of plasmapause extent on ground-based observations of chorus via a 3 month study of ground-based measurements of chorus at Palmer Station, Antarctica $\left(L=2.4,50^{\circ} \mathrm{S}\right.$ geomagnetic latitude), and data on the plasmapause extent from the IMAGE EUV instrument. It is found that chorus normalized occurrence peaks when the plasmapause is at $L \sim 2.6$, somewhat higher than Palmer's $L$ shell, and that this occurrence peak persists across a range of observed chorus frequencies. Next, reverse ray tracing is employed to evaluate the portion of the equatorial chorus source region, distributed in radial distance and wave normal, from which chorus is able to reach Palmer Station via propagation in a nonducted mode. The results of ray tracing are similar to those of observations, with a peak of expected occurrence when the plasmapause is at $L \sim 3$. The exact location of the peak is frequency dependent. This supports the conclusion that the ability of chorus to propagate to low altitudes and the ground is a strong function of instantaneous plasmapause extent and that peak occurrence of chorus at a given ground station may occur when the $\mathrm{L}$ shell of the plasmapause is somewhat beyond that of the observing station. These results also suggest that chorus observed on the ground at midlatitude stations propagates predominantly in the nonducted mode.

Citation: Golden, D. I., M. Spasojevic, F. R. Foust, N. G. Lehtinen, N. P. Meredith, and U. S. Inan (2010), Role of the plasmapause in dictating the ground accessibility of ELF/VLF chorus, J. Geophys. Res., 115, A11211, doi:10.1029/2010JA015955.

\section{Introduction}

[2] Extremely low frequency/very low frequency (ELF/ VLF) chorus emissions are electromagnetic waves which are spontaneously generated in the Earth's magnetosphere. Chorus is characterized as consisting of repeating, usually rising and often overlapping coherent tones and is often accompanied by a band of hiss [e.g., Cornilleau-Wehrlin et $a l ., 1978]$. In recent years, chorus has received increased attention due to the role that it is thought to play in the acceleration [e.g., Meredith et al., 2002; Horne et al., 2003, 2005] and loss [e.g., Lorentzen et al., 2001; O'Brien et al., 2003; Thorne et al., 2005; Shprits et al., 2006] of energetic electrons in the Earth's radiation belts. Additionally, some fraction of chorus may act via its evolution into plasmaspheric hiss [Parrot et al., 2004; Santolik et al., 2006; Bortnik et al., 2008] as an additional loss agent for energetic

\footnotetext{
${ }^{1}$ STAR Laboratory, Stanford University, Stanford, California, USA.

${ }^{2}$ British Antarctic Survey, Natural Environment Research Council, Cambridge, UK.

${ }^{3}$ Electrical Engineering Department, Koç University, Istanbul, Turkey.

Copyright 2010 by the American Geophysical Union. 0148-0227/10/2010JA015955
}

electrons [e.g., Lyons et al., 1972; Lyons and Thorne, 1973; Abel and Thorne, 1998; Meredith et al., 2007].

[3] Chorus waves are believed to be generated by a Doppler-shifted cyclotron interaction between anisotropic distributions of energetic $>40 \mathrm{keV}$ electrons and ambient background VLF noise [Tsurutani and Smith, 1974, 1977; Thorne et al., 1977]. These unstable distributions can result from substorm injection, and correspondingly, chorus is predominantly observed across the morning and noon local time sectors in association with eastward drifting electrons. Because magnetic substorms both increase the flux of hot source electrons which generate chorus as well as enhance the auroral electrojet (AE), increases in the $\mathrm{AE}$ index have been shown to be a good predictor of chorus occurrence within the inner magnetosphere [Smith et al., 1999; Meredith et al., 2001]. The outer dayside region of the magnetosphere is also conducive to chorus generation, but here waves are less dependent on substorm activity and can be observed under both quiet and disturbed geomagnetic conditions [Tsurutani and Smith, 1977; Li et al., 2009; Spasojevic and Inan, 2010].

[4] Ground-based measurements of ELF/VLF emissions are by definition limited to the small subset of space-based 
emissions that are able to penetrate to low altitudes and through the ionosphere [e.g., Sonwalkar, 1995, pp. 424 425]. Ground-based observations may include (1) waves that have propagated such that their wave normals naturally arrive within the transmission cone at the ionospheric boundary [Helliwell, 1965, section 3.7], (2) waves that have propagated within field-aligned density irregularities known as "ducts" [e.g., Smith, 1961; Carpenter, 1966; Carpenter and Sulic, 1988], which have the effect of constraining the wave normals to be nearly field aligned, or (3) waves that arrive at the ionospheric boundary with nonvertical wave normals and are then scattered from low-altitude meter-scale density irregularities [Sonwalkar and Harikumar, 2000] that rotate the wave normals into the transmission cone.

[5] In situ measurements of chorus have shown that chorus occurs in two bands, separated by half the equatorial gyrofrequency $\left(f_{c e q}\right)$ along the observation field line [Tsurutani and Smith, 1974; Burtis and Helliwell, 1976; Tsurutani and Smith, 1977]. Of the two bands, only the lower band is thought to reach the ground; the upper band is believed to reflect at high altitudes due to its highly oblique wave normal angle [Hayakawa et al., 1984; Haque et al., 2010]. Thus, chorus received on the ground is expected to be exclusively lower band chorus, generated below half the equatorial gyrofrequency.

[6] The current work is motivated by a recent statistical study by Golden et al. [2009] of chorus and hiss observed on the ground at Palmer Station, Antarctica, at $L=2.4,50^{\circ} \mathrm{S}$ geomagnetic latitude. During the course of that study, which spanned 10 months in 2003, chorus was observed on more than $50 \%$ of days. This was unexpected for several reasons. First, chorus is generated outside the plasmasphere, according to early satellite studies [e.g., Gurnett and O'Brien, 1964; Dunckel and Helliwell, 1969] which have shown that chorus is most commonly observed outside the plasmasphere. In addition, chorus observed on the ground has traditionally been interpreted as a ducted emission, and therefore, that the L shell on which it is received is approximately the same as the $\mathrm{L}$ shell on which it is generated. The presumption that nonducted chorus cannot penetrate to the ground [e.g., Imhof et al., 1989, p. 10,092] is based on ray tracing results that show that nonducted whistlers will magnetospherically reflect before returning to the ground [Kimura, 1966; Edgar, 1976] and is supported by occasional observation of chorus-like noise bursts that, in ground observations, appear to have been triggered [Carpenter et al., 1975] or damped [Gail and Carpenter, 1984] by ducted whistlers (implying that the observed whistlers and chorus share the same duct). However, in the study of Golden et al. [2009], the magnetospheric conditions were such that the plasmapause was often expected to be well beyond Palmer's L shell during chorus observations. During that study, chorus was observed for $\mathrm{Kp} \lesssim 2^{+}$. According to the plasmapause model of Carpenter and Anderson [1992], at $\mathrm{Kp}=2^{+}$, the plasmapause is expected to be around $L \sim 4.5$. It is only for $\mathrm{Kp}>6^{+}$that the plasmapause is expected to reach down to $L<2.5$. Also, the frequency range of observed chorus suggests that the source region of the waves is well beyond Palmer's L shell. Satellite studies have shown that lower band chorus is generated for frequencies in the range $0.1 f_{\text {ceq }} \leq f \leq 0.5 f_{\text {ceq }}$ [Tsurutani and Smith, 1974; Burtis and Helliwell, 1976]. Waves of frequencies below $500 \mathrm{~Hz}$ were observed by Golden et al. [2009], which corresponds to a source location of $L>5.5$ under a dipole model of the Earth's magnetic field.

[7] It seems clear that the observations of Golden et al. [2009] are inconsistent with the theory of ducted propagation of chorus and that the dominant mode of chorus reception at midlatitude stations like Palmer may instead be nonducted. In support of this possibility, Chum and Santolik [2005] have shown via ray tracing that nonducted chorus, generated in the equatorial magnetosphere with wave normal angles near the local Gendrin angle, may be able to reach the ionosphere and penetrate to the ground at L shells significantly below those at which the waves are generated. Although Chum and Santolik [2005] did not include a plasmasphere in their analysis, it seems logical, given the exoplasmaspheric source of chorus and the location of Palmer within the plasmasphere, that the location of the plasmapause may play an important role in determining which subsets of chorus may be able to be received at Palmer.

[8] In this study, we address two broad questions. (1) What is the location of the plasmapause when chorus is observed at Palmer? (2) How does the location of the plasmapause affect the portion of the chorus source region that is able to propagate to the ground and be received at Palmer? These questions are answered via a combination of (1) a 3 month statistical study of chorus observations using the Stanford ELF/VLF wave receiver at Palmer Station coupled with simultaneous measurements of the plasmapause using the Extreme Ultraviolet (EUV) instrument on board the IMAGE satellite and (2) a model-based study of chorus propagation effects via a new Stanford VLF 3-D ray tracing software package, used to model magnetospheric propagation and Landau damping under different models of the plasmapause location, as well as a full wave code, used to model electromagnetic propagation in the Earth-ionosphere waveguide.

\section{Experimental Methodology}

[9] In order to determine the location of the plasmapause when chorus is observed at Palmer Station, we employ two separate databases: a database of emissions observed at Palmer Station and a database of plasmapause locations at Palmer's MLT. Both databases span 3 months, from April through June 2001, and are discussed below.

\subsection{Palmer Emission Database}

[10] Palmer Station is located on Anvers Island, near the tip of the Antarctic peninsula, at $64.77^{\circ} \mathrm{S}, 64.05^{\circ} \mathrm{W}$, with IGRF geomagnetic parameters of $L=2.4,50^{\circ} \mathrm{S}$ geomagnetic latitude, and magnetic local time $(\mathrm{MLT})=\mathrm{UTC}-4.0$ at $100 \mathrm{~km}$ altitude. The Palmer VLF receiver records broadband VLF data at 100 kilosamples per second using two cross-loop magnetic field antennas, with $96 \mathrm{~dB}$ of dynamic range. This analysis uses the North/South channel exclusively, it being the less subjectively noisy of the two channels; this has the additional effect of focusing Palmer's viewing area more tightly to its magnetic meridian than if both channels were used. Data products used in this study are $10 \mathrm{~s}$ broadband data files, subsampled at a rate of 20 kilosamples per second, beginning every $15 \mathrm{~min}$ at $5,20,35$, and $50 \mathrm{~min}$ past the hour, $24 \mathrm{~h}$ per day. 


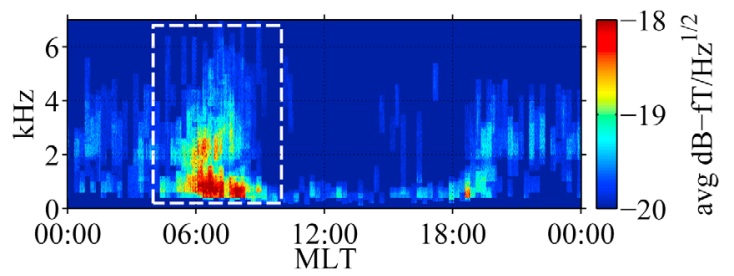

Figure 1. Cumulative spectrogram of chorus emissions from April through June 2001. Only emissions in the boxed area, between 04 and 10 MLT, are used in this study.

[11] The year 2001 falls approximately on the peak of Solar Cycle 23, and chorus occurrence is frequent at Palmer Station during this period. A combination of automated emission detection (D. I. Golden and M. Spasojevic, Determination of solar cycle variations of midlatitude ELF/VLF chorus and hiss via automated signal detection, submitted to Journal of Geophysical Research, 2010) and manual correction is used to determine the presence of emissions. The automated detector rejects confounding impulsive electromagnetic signals, such as sferics and whistlers, and focuses on chorus and hiss. Chorus is then distinguished from hiss based on its "burstiness," namely, the frequency content of the amplitude modulation of the broadband signal. Bursty signals are classified as chorus, and nonbursty signals are classified as hiss, and discarded. The output of the automated detector is then manually verified to eliminate false positives (e.g., hiss or lightning-generated whistlers erroneously labeled as chorus) and false negatives (e.g., weak chorus emissions that may have been rejected based on their proximity to sferics or other emissions). Although it is likely that some chorus emissions with low signal-to-noise ratios are erroneously rejected by this algorithm, the profusion of detected chorus emissions still leads to statistically significant results.

[12] We define a "synoptic epoch" as an interval during which Palmer data is sampled for this study. Each universal hour contains four synoptic epochs, at 5, 20, 35, and $50 \mathrm{~min}$ past the hour. At each synoptic epoch, a binary judgment is made about whether chorus is observed or not, based on the results of both the automated detector and manual inspection. The resulting table of true/false values for chorus observation versus time then becomes the database of Palmer chorus emissions. As an overview, Figure 1 shows a cumulative spectrogram of the chorus emissions used in this study. The cumulative spectrogram is effectively the logarithmic sum of the spectrums of its constituent emissions, and is a measure of the average chorus spectrum with respect to frequency and local time. The full procedure is described by Golden et al. [2009, section 2.2]. The gap at $\sim 1.7 \mathrm{kHz}$ on the cumulative spectrogram is a result of increased attenuation below the first transverse electric $\left(\mathrm{TE}_{1}\right)$ waveguide mode cutoff during propagation in the Earthionosphere waveguide. Only emissions in the boxed region, in the range $4 \leq$ MLT $\leq 10$ are used in this study.

\subsection{Plasmapause Location Database}

[13] In order to determine the instantaneous plasmapause location at each synoptic epoch, data from the Extreme
Ultraviolet (EUV) instrument [Sandel et al., 2000] on board the IMAGE satellite [Burch, 2000] are used. The EUV instrument images resonantly scattered sunlight from $\mathrm{He}^{+}$ ions, which are a minority constituent of the plasma in the Earth's plasmasphere. The $\mathrm{He}^{+}$edge, as seen by the EUV instrument, has been shown to be an accurate proxy for the plasmapause [Goldstein et al., 2003], which is the region of the magnetosphere where the electron density exhibits a steep drop with increasing $L$ value.

[14] Because this study focuses on emissions observed on the ground at Palmer, the extent of the plasmapause is only considered at Palmer's magnetic local time, MLT $=$ UTC 4.0. Raw EUV images are initially mapped to the equatorial plane using the minimum L technique of Roelof and Skinner [2000, section 2.2], assuming a dipole model for the Earth's magnetic field. The radial extent of the plasmapause is then manually selected on each individual EUV image at MLT = UTC -4.0 and that plasmapause value is added to the database. EUV images where the plasmapause cannot be found due to excessive noise or EUV camera malfunction, or where the plasmapause is either poorly defined or not visible below $L=6$, are discarded. After removing data gaps from both databases, 1033 synoptic epochs, or approximately $260 \mathrm{~h}$ of data, remain for this study.

\section{Dependence of Chorus Observations on Plasmapause Extent}

\subsection{Choice of AE Metric}

[15] Since this study concerns the role of the plasmapause in dictating the observation of chorus emissions, it is instructive to make mention of how the plasmapause is correlated with the $\mathrm{AE}$ index, which is itself well correlated with the observation of chorus emissions [e.g., Meredith et al., 2001]. This is done to explore a potential confounding effect where a single event, namely a magnetic substorm, may have two simultaneous consequences: (1) enhancement of the auroral electrojet, causing an increase in AE, and (2) erosion of the plasmasphere.

[16] Figure 2 shows the extent of the plasmapause, sampled at $04 \leq \mathrm{MLT} \leq 10, \mathrm{MLT}=\mathrm{UTC}-4.0$, plotted against the instantaneous $\mathrm{AE}$ index (left), and the average $\mathrm{AE}$ in the

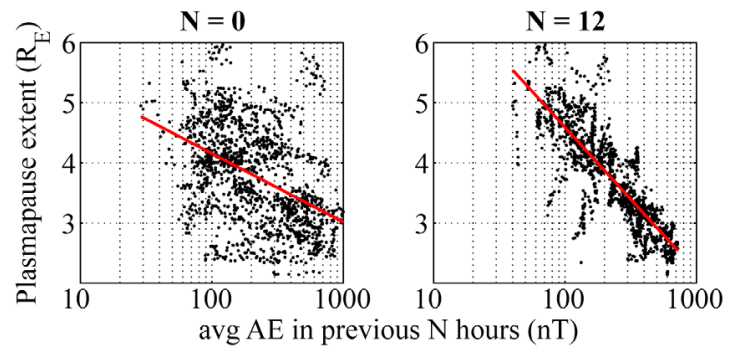

Figure 2. $\mathrm{L}$ shell of plasmapause at MLT $=\mathrm{UTC}-4.0$ within the range $04 \leq$ MLT $\leq 10$ plotted against (left) instantaneous $\mathrm{AE}$ and (right) average $\mathrm{AE}$ in the previous 12 hours. Plasmapause extent is moderately correlated with instantaneous $\operatorname{AE}\left(\rho=-0.43, \sigma_{\text {err }}=0.75 L\right)$ and highly correlated with average $\mathrm{AE}$ in the previous 12 hours $(\rho=-0.81$, $\sigma_{\text {err }}=0.49 L$ ). In each plot, the solid red line is a linear fit between plasmapause $L$ and the logarithm of AE. 


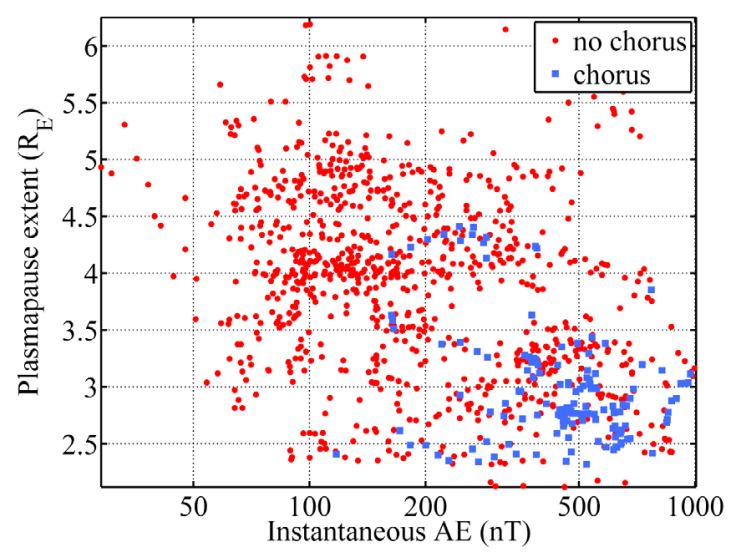

Figure 3. Scatterplot of synoptic epochs with (blue squares) and without (red dots) chorus. Note that AE is displayed on a logarithmic scale, while plasmapause extent is displayed on a linear scale.

previous 12 hours (right), over the 3 month period of this study. Averaging the AE index over $N=12$ hours yields approximately the greatest correlation for any value of $N$. The plasmapause is moderately correlated with the $\log$ of instantaneous $\mathrm{AE}$, with correlation coefficient $\rho=-0.43$ and residual standard deviation $\sigma_{\text {err }}=0.75 \mathrm{~L}$, and highly correlated with the log of the average $\mathrm{AE}$ in the previous 12 hours, with correlation coefficient $\rho=-0.81$ and residual standard deviation $\sigma_{\text {err }}=0.49 \mathrm{~L}$.

[17] However, the manner in which AE is associated with plasmapause extent differs from how it is expected to be associated with chorus occurrence. The time between when $\mathrm{AE}$ is enhanced and when chorus is expected to be seen at Palmer may be determined by calculating the expected time required for a chorus source particle to drift from 00 MLT to 06 MLT. Based on work by Walt [1994, Figure B.2], $100 \mathrm{keV}$ electrons at $L=4$ will drift from midnight to 06 MLT in $21 \mathrm{~min}$; higher-energy particles will drift more quickly. This time period is on the order of the synoptic epoch used in this study (15 min). Therefore instantaneous $\mathrm{AE}$ is used as the metric for predicting chorus in this study. It is significant that, while instantaneous $\mathrm{AE}$ is expected to be a good predictor of chorus occurrence, it is only weakly correlated with plasmapause extent. This suggests that source effects, as measured by instantaneous $\mathrm{AE}$, and propagation effects, as measured by plasmapause extent, may exert independent control over the probability that chorus will be seen at Palmer at any given time.

\subsection{Chorus Occurrence Versus Plasmapause Extent}

[18] Here the dependence of chorus normalized occurrence on plasmapause extent is examined. The additional complication of $\mathrm{AE}$ is deferred to the multivariate analysis of section 3.3. Although the detailed structure of the plasmapause boundary layer is complex [Carpenter and Lemaire, 2004], the major plasmapause structure is assumed to be field aligned over much of its range. For the purposes of this study, the plasmapause can therefore be described via the scalar quantity $L_{\mathrm{PP}}$, which represents the equatorial plasmapause extent, in units of Earth radii. A scatterplot of chorus observations at each synoptic epoch versus instantaneous $\mathrm{AE}$ and $L_{\mathrm{PP}}$ is shown in Figure 3. Synoptic epochs with chorus are indicated with blue squares and epochs without chorus are indicated with red dots. The scattered points themselves are the same as in the left panel of Figure 2, with some data gaps removed. One can get the general impression from this plot that chorus is more likely to be observed at Palmer for low $L_{\mathrm{PP}}$ and high AE. To examine the data more rigorously, regression analysis is used to construct a generalized linear model [e.g., Chatterjee and Hadi, 2006] of chorus normalized occurrence as a function of plasmapause extent. This provides additional insight into properties that are not obvious from a simple scatterplot, such as at which $L_{\mathrm{PP}}$ chorus occurrence is maximized, and how strong that peak is.

[19] Under regression analysis, a linear combination of parameters is sought to form an estimate of $\mu$, the probability of chorus occurrence. Because linear models have, in general, unbounded values, a logit response function is used for $\mu$, defining the output of the linear model, $Y$, as

$$
Y=\log \left(\frac{\mu}{1-\mu}\right)
$$

and, conversely,

$$
\mu=\frac{e^{Y}}{1+e^{Y}} .
$$

This transforms the bounded parameter $\mu \in[0,1]$ to the unbounded parameter $Y \in(-\infty, \infty)$. Given $p$ distinct independent variables, $Y$ is modeled as

$$
Y=X \beta=\left[\begin{array}{lllll}
1, & x_{1}, & x_{2}, & \ldots, & x_{p}
\end{array}\right]\left[\begin{array}{c}
\beta_{0} \\
\beta_{1} \\
\beta_{2} \\
\vdots \\
\beta_{p}
\end{array}\right],
$$

where $X$ is a row vector of predictors, formed by transformations of the independent variables (e.g., $x_{1}=L_{\mathrm{PP}}, x_{2}=$ $L_{\mathrm{PP}}^{2}$, etc.), and $\beta$ is a column vector of coefficient estimates.

[20] The generalized linear model regression procedure from the MATLAB software package is used to obtain a linear fit. Although it is possible to include an arbitrary number of powers of $L_{\mathrm{PP}}$ in the model, we honor the principle of parsimony and favor simpler models. Bayesian Information Criterion (BIC) [Chatterjee and Hadi, 2006, section 12.6] is employed for this purpose, which assigns any particular model a lower score for better goodness of fit, and a higher score for each included term; lower scores are favored. Additionally, the maximum model order is restricted to 4 .

[21] To determine whether there is any frequency dependence in the degree to which chorus occurrence changes with $L_{\mathrm{PP}}$, the regression analysis is separately performed on three cases: all frequencies, $f<1.5 \mathrm{kHz}$ and $f>3 \mathrm{kHz}$. For all frequencies and $f<1.5 \mathrm{kHz}$, the fourth-order model has the lowest BIC and is therefore the favored model. For $f>3 \mathrm{kHz}$, the second-order model has the lowest BIC. The model parameters for the three cases, along with the $\mathrm{p}$ values, are 
Table 1. Univariate Model Coefficients

\begin{tabular}{lcc}
\hline$X$ & $\beta$ & $\mathrm{p}$ Value \\
\hline & All Frequencies & \\
1 & -389 & $1.78 \times 10^{-5}$ \\
$L_{\mathrm{PP}}$ & 465 & $2.33 \times 10^{-5}$ \\
$L_{\mathrm{PP}}^{2}$ & -204 & $3.47 \times 10^{-5}$ \\
$L_{\mathrm{PP}}^{3}$ & 38.7 & $5.77 \times 10^{-5}$ \\
$L_{\mathrm{PP}}^{4}$ & -2.71 & $9.86 \times 10^{-5}$ \\
& & \\
1 & $f<1.5 \mathrm{kHz}$ & \\
$L_{\mathrm{PP}}$ & -417 & $1.36 \times 10^{-5}$ \\
$L_{\mathrm{PP}}^{2}$ & 503 & $1.53 \times 10^{-5}$ \\
$L_{\mathrm{PP}}^{3}$ & -223 & $1.98 \times 10^{-5}$ \\
$L_{\mathrm{PP}}^{4}$ & 42.8 & $2.94 \times 10^{-5}$ \\
& -3.02 & $4.64 \times 10^{-5}$ \\
& & \\
1 & $f>3 \mathrm{kHz}$ & $2.15 \times 10^{-3}$ \\
$L_{\mathrm{PP}}$ & -35.8 & $1.76 \times 10^{-3}$ \\
$L_{\mathrm{PP}}^{2}$ & 25.8 & $8.99 \times 10^{-4}$ \\
\hline
\end{tabular}

shown in Table 1. The $\mathrm{p}$ value in this case represents the probability of erroneously assigning a nonzero value to a given coefficient when its true value is zero. Since all of the $\mathrm{p}$ values are well below 0.05 , we can safely assume that all coefficients are significant.

[22] Figure 4 shows the modeled normalized occurrence as a function of plasmapause extent for the three cases of all frequencies (left), $f<1.5 \mathrm{kHz}$ (center) and $f>3 \mathrm{kHz}$ (right). $\mu$ is indicated by a solid black line, and the $95 \%$ confidence intervals of the fit are indicated by the surrounding shaded regions. The model for $f<1.5 \mathrm{kHz}$ is quite similar to the one for all frequencies, with the same predictors $X$ and similar coefficients $\beta$. The model for $f>3 \mathrm{kHz}$ is rather different, with different $X$. This is a consequence of the fact that $80 \%$ of chorus observed at Palmer includes frequency compo-

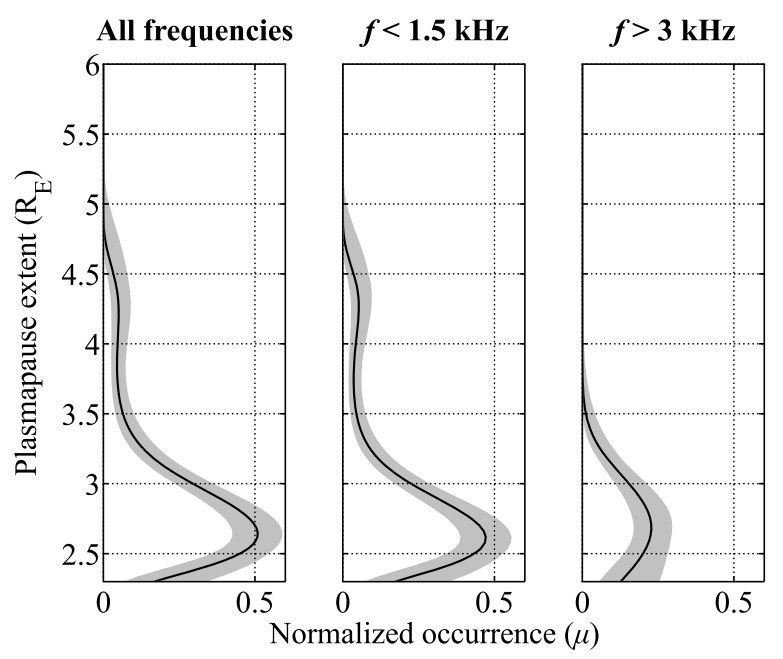

Figure 4. Predicted normalized occurrence rate $(\mu)$ as a function of plasmapause extent $\left(L_{\mathrm{PP}}\right)$ for chorus observations at Palmer for (left) all frequencies, (center) $f<1.5 \mathrm{kHz}$, and (right) $f>3 \mathrm{kHz}$. Solid lines indicate modeled values of $\mu$, and shaded regions indicate $95 \%$ confidence intervals. The models for all frequencies and $f<1.5 \mathrm{kHz}$ include up to fourth-order terms of $L_{\mathrm{PP}}$, while the model for $f>3 \mathrm{kHz}$ includes up to second-order terms.
Table 2. Bivariate Model Coefficients (All Frequencies)

\begin{tabular}{lcc}
\hline \multicolumn{1}{c}{$X$} & \multicolumn{1}{c}{$\beta$} & $\mathrm{p}$ Value \\
\hline 1 & $1.053 \times 10^{3}$ & $9.4 \times 10^{-5}$ \\
$\log _{10} \mathrm{AE}$ & $-1.850 \times 10^{3}$ & $1.4 \times 10^{-5}$ \\
$\log _{10} \mathrm{AE} \cdot L_{\mathrm{PP}}$ & $6.356 \times 10^{2}$ & $1.9 \times 10^{-6}$ \\
$\left(\log _{10} \mathrm{AE}\right)^{2}$ & $7.642 \times 10^{2}$ & $9.1 \times 10^{-6}$ \\
$L_{\mathrm{PP}}^{2}$ & $-2.316 \times 10^{2}$ & $3.4 \times 10^{-6}$ \\
$\left(\log _{10} \mathrm{AE}\right)^{2} \cdot L_{\mathrm{PP}}$ & $-2.588 \times 10^{2}$ & $1.8 \times 10^{-6}$ \\
$\left(\log _{10} \mathrm{AE}\right)^{3}$ & $-1.042 \times 10^{2}$ & $7.5 \times 10^{-6}$ \\
$L_{\mathrm{PP}}^{3}$ & $4.518 \times 10^{1}$ & $5.5 \times 10^{-6}$ \\
$\left(\log _{10} \mathrm{AE}\right)^{3} \cdot L_{\mathrm{PP}}$ & $3.486 \times 10^{1}$ & $2.0 \times 10^{-6}$ \\
$L_{\mathrm{PP}}^{4}$ & $-3.245 \times 10^{0}$ & $9.9 \times 10^{-6}$ \\
\hline
\end{tabular}

nents below $1.5 \mathrm{kHz}$, but only $33 \%$ of chorus includes components above $3 \mathrm{kHz}$.

[23] A distinct feature of all curves is a "saturation" effect, where chorus occurrence does not increase monotonically with decreasing plasmapause extent; instead, a peak in occurrence can be seen at $L_{\mathrm{PP}}=2.6$ for $f<1.5 \mathrm{kHz}$ and at $L_{\mathrm{PP}}=2.7$ for $f>3 \mathrm{kHz}$. Additionally, the curve for $f<$ $1.5 \mathrm{kHz}$ has a longer tail for higher $L_{\mathrm{PP}}$ than that of $f>$ $3 \mathrm{kHz}$, indicating that a less disturbed (more extended) plasmasphere permits only lower-frequency chorus access to Palmer.

\subsection{Chorus Occurrence Versus Plasmapause Extent and $\mathbf{A E}$}

[24] Although it was shown in section 3.2 that plasmapause extent is strongly related to chorus normalized occurrence at Palmer, it is not yet clear whether this is truly a consequence of the instantaneous plasmapause extent or whether it is simply a consequence of the fact that magnetic substorms both increase the likelihood of chorus and, separately, cause erosion of the plasmapause. To explore this confounding effect, multiple regression is used to separately examine dependence of $\mu$ on both plasmapause extent, which may affect chorus propagation, and AE, which is related to chorus generation.

[25] Again, a solution to (3) is sought, except that now $X$ includes $L_{\mathrm{PP}}$ and $\log _{10} \mathrm{AE}$ terms as well as interaction terms. Beginning with a model that includes all permutations of $L_{\mathrm{PP}}$ through $L_{\mathrm{PP}}^{4}$ and $\log _{10} \mathrm{AE}$ through $\left(\log _{10} \mathrm{AE}\right)^{4}$ of total order 4 or less, terms with high $\mathrm{p}$ values whose removal increases BIC are dropped. Eventually, the model of Table 2 is found. Table 2 shows the selected model parameters, their coefficients, and the $\mathrm{p}$ value of each coefficient.

[26] A plot of $\mu$, the modeled parameter of (2), as a function of $L_{\mathrm{PP}}$ and $\log _{10} \mathrm{AE}$ for all frequencies, is shown in Figure 5a. To reduce noise in Figure 5a, the actual plotted quantity is $\mu \cdot\left(1-\sigma_{95}^{2}\right)$ instead of $\mu$, where $\sigma_{95}$ is the range of the $95 \%$ confidence interval, obtained by subtracting Figure $5 \mathrm{c}$ from Figure $5 \mathrm{~b}$. This has the effect of setting areas with high variance to zero, e.g., the lower left and upper right portions of the plot. As in section 3.2, a saturation effect is seen with respect to $L_{\mathrm{PP}}$, and a peak in $\mu$ is seen at $L_{\mathrm{PP}}=2.6$ for $\mathrm{AE} \gtrsim 100 \mathrm{nT}$. Additionally, the long tail in $L_{\mathrm{PP}}$ is reproduced, with $\mu$ retaining a small but nonzero value up to $L_{\mathrm{PP}} \sim 4.5$.

[27] The primary takeaway fact from Figure 5 is that features with respect to $L_{\mathrm{PP}}$ persist for a wide range of $\mathrm{AE}$, and features with respect to $\mathrm{AE}$ persist for a wide range of $L_{\mathrm{PP}}$. For example, the peak at $L_{\mathrm{PP}}=2.6$ exists for $200 \mathrm{nT} \lesssim$ 
Normalized occurrence $(\mu$, mean) $\quad \mu$, upper 95\% C.I.

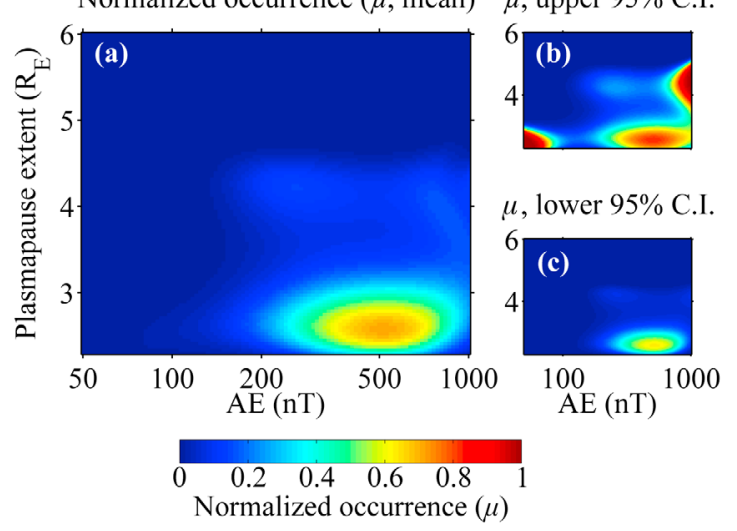

Figure 5. Model for $\mu$, the normalized occurrence rate of chorus as a function of plasmapause extent and AE, obtained using generalized linear model regression on observations of chorus. (a) The expected value of normalized occurrence. (b) Upper and (c) lower bounds of the $95 \%$ confidence interval for $\mu$.

$\mathrm{AE} \lesssim 1000 \mathrm{nT}$, and the peak at $\mathrm{AE}=500 \mathrm{nT}$ exists for $2.1 \lesssim L_{\mathrm{PP}} \lesssim 3.1$. This is an indication that effects of $\mathrm{AE}$ or $L_{\mathrm{PP}}$ near the peak of chorus occurrence are quasi-independent of each other. Had it been otherwise, and the effects of AE and $L_{\mathrm{PP}}$ were strongly dependent, the peak in Figure 5 would appear as a diagonal line. Therefore, it is clear that the plasmapause is in fact significantly changing the characteristics of chorus propagation to Palmer, and that the correlation between $L_{\mathrm{PP}}$ and $\mu$ is not merely a confounding effect of the fact that magnetic substorms tend to affect both chorus generation and the plasmapause.

\section{Modeling of Chorus Propagation}

[28] The effects of plasmapause extent on chorus propagation are further investigated using a combination of ray tracing and full wave modeling. First, reverse ray tracing is used wherein rays begin above the ionosphere over Palmer with wave normal angles within the ionospheric transmission cone. The rays are then propagated backward to their magnetospheric source. A valid source location for each ray is outside the plasmasphere at the magnetic equatorial plane [LeDocq et al., 1998; Santolik et al., 2005] at a radial distance such that the wave frequency is in the range 0.1 $f_{\text {ceq }} \leq f \leq 0.5 f_{\text {ceq }}$ [Tsurutani and Smith, 1974; Burtis and Helliwell, 1976]. Rays that are able to enter a valid source location are binned by radial extent and wave normal angle. This creates a comprehensive picture of the portion of the equatorial source region from which generated rays may reach Palmer. Ray attenuation is calculated via Landau damping on the magnetospheric ray paths using an empirical model of energetic particle fluxes. In addition, we assume that waves may penetrate the ionosphere some distance from Palmer and propagate within the Earth-ionosphere waveguide before being received; a full wave model is used to estimate this additional waveguide attenuation. Full details of the simulation are further discussed below. The simulation is performed for a range of plasmapause extents. For each plasmapause extent, a single scalar quantity is calculated, which we term the Chorus Availability Factor (CHAF). $\mathrm{CHAF}$ is a cumulative measure of the portion of the chorus source region, integrated over all radial extents and wave normals, and weighted by relative attenuation and source probability, that is observable at Palmer. Although CHAF is not a probability, if the plasmapause extent does significantly influence chorus propagation, the trends of CHAF versus $L_{\mathrm{PP}}$ are expected to resemble those of the experimentally modeled chorus normalized occurrence, $\mu$, from section 3.2.

\subsection{Stanford VLF 3-D Ray Tracer}

[29] The new version of the Stanford VLF ray tracer was developed by one of us (F. R. F.) as a more accurate and complete model to replace Stanford's previous ray tracing program [Inan and Bell, 1977], which we refer to as the Stanford VLF legacy ray tracer. The new ray tracer, which we refer to as the Stanford VLF 3-D ray tracer, was written from the ground up, and is not an extension or revision of the Stanford VLF legacy ray tracer. A description of the ray tracer follows.

[30] Hamilton's equations for the propagation of a ray through a medium with spatially varying dispersion relation defined by the implicit function $F(\omega, \mathbf{k}, \mathbf{r})=0$ can be stated as

$$
\begin{gathered}
\frac{d \mathbf{r}}{d t}=-\frac{\nabla_{k} F}{\partial F / \partial \omega} \\
\frac{d \mathbf{k}}{d t}=\frac{\nabla_{r} F}{\partial F / \partial \omega}
\end{gathered}
$$

with the constraint

$$
F(\omega, \mathbf{k}, \mathbf{r})=0
$$

For generality, and for the purpose of accommodating any arbitrary function for the plasma density or background magnetic field, the spatial and $\mathrm{k}$-space derivatives are evaluated numerically using finite differences; that is,

$$
\begin{aligned}
& \frac{\partial F}{\partial k_{i}} \approx \frac{1}{2 \Delta k}\left(F\left(\omega, \mathbf{k}+\Delta k \mathbf{e}_{i}, \mathbf{r}\right)-F\left(\omega, \mathbf{k}-\Delta k \mathbf{e}_{i}, \mathbf{r}\right)\right) \\
& \frac{\partial F}{\partial r_{i}} \approx \frac{1}{2 \Delta r}\left(F\left(\omega, \mathbf{k}, \mathbf{r}+\Delta r \mathbf{e}_{i}\right)-F\left(\omega, \mathbf{k}, \mathbf{r}-\Delta r \mathbf{e}_{i}\right)\right),
\end{aligned}
$$

where $i=\{1,2,3\}$, and $\mathbf{e}_{i}$ are the unit vectors. Since the derivatives are evaluated numerically, all that is required to adapt a new plasma density model is a function that evaluates $F(\omega, \mathbf{k}, \mathbf{r})$.

[31] After approximating the spatial and k-space derivatives, six ordinary differential equations remain, which are integrated numerically in time using a standard adaptive Runge-Kutta method. In contrast to the approach of Haselgrove [1955], a moving $B_{0}$-aligned coordinate system is not used; instead, the system of equations is directly solved in global Cartesian coordinates. After one time step, the constraint $F=0$ is not in general met, and an intermediate solution exists with an error $F\left(\omega, \mathbf{k}^{*}, \mathbf{r}^{*}\right)=\epsilon$. This is handled using a standard method for solving constrained ODEs, by 
finding a "nearby" point $(\mathbf{k}, \mathbf{r})$ that satisfies $F(\omega, \mathbf{k}, \mathbf{r})=0$ after every time step. The specific approach used is to simply re-solve the dispersion relation assuming the wave normal angle is kept constant. If this fails (due to being too close to the resonance cone), the time step is halved and the procedure is attempted again.

[32] The Stanford VLF 3-D ray tracer can accommodate any arbitrary function for the cold background plasma number density. In this study, the Global Core Plasma Model (GCPM) [Gallagher et al., 2000] is implemented, sampled on a regular grid and interpolated by a fast, local, $C^{1}$ (continuous in the first derivative) tricubic interpolation scheme described by Lekien and Marsden [2005]. The plasmasphere modeled by the GCPM is field aligned to the dipole field, and remains so from the equatorial region down to altitudes between $7800 \mathrm{~km}\left(\mathrm{~K}_{\mathrm{p}} \sim 3^{+}\right)$to $2600 \mathrm{~km}\left(\mathrm{~K}_{\mathrm{p}} \sim 8^{-}\right)$. The typical plasmapause represented by the GCPM exhibits a density drop of between $1\left(\mathrm{~K}_{\mathrm{p}} \sim 3^{+}\right)$and $1.5\left(\mathrm{~K}_{\mathrm{p}} \sim 8^{-}\right)$orders of magnitude in the equatorial plane over a range of about $0.3 \mathrm{R}_{\mathrm{E}}$. The choice of background magnetic field is also arbitrary; in this study, the Tsyganenko-96 (T96) model [Tsyganenko, 1995; Tsyganenko and Stern, 1996] is used.

[33] Thermal losses are included as in work by Kennel [1966]. Equation (3.9) by Kennel [1966], corrected for a typographical error [Chen et al., 2009, paragraph 9], is solved for the Landau $(m=0)$ resonance. This yields the temporal damping rate $\omega_{i}$, which is then related to the spatial damping rate $k_{i}$ by the relation by Brinca [1972]:

$$
\omega_{i}=\overrightarrow{k_{i}} \cdot \overrightarrow{v_{g}}
$$

The method by Kennel [1966] requires the evaluation of the gradients of the hot particle distribution function in $\left(v_{\|}, v_{\perp}\right)$ space, as well as the evaluation of a 1-D integral over $v_{\perp}$ over the interval $[0, \infty)$. In order to accommodate any arbitrary distribution function, the derivatives are again evaluated numerically using finite differences. The velocity is first normalized by the speed of light for numerical reasons, then mapped into a finite range $t=(0,1)$ using the mapping $v_{\perp}=(1-t) / t$ :

$$
\int_{0}^{\infty} f\left(v_{\perp}\right) d v_{\perp}=\int_{0}^{1} \frac{1}{t^{2}} f\left(\frac{1-t}{t}\right) d t .
$$

Finally, the integral is evaluated numerically using adaptive quadrature. The method used is general and can accommodate any number of resonances. In this study, only the Landau $(m=0)$ resonance is used, since it is the dominant source of loss.

[34] The choice of hot particle distribution is crucial to the accurate calculation of Landau damping. Within the plasmasphere, the phase space density expression of Bell et al. [2002], based on measurements with the POLAR spacecraft sampled in the range $2.3<L<4$, is used. Outside the plasmasphere, the methodology of Bortnik et al. [2007a], derived from measurements with the CRRES spacecraft outside the plasmasphere up to $L \sim 7$, is used.

[35] A hybrid model smooths the two models at the plasmasphere boundary, and is implemented as follows. Let $f_{0}^{\mathrm{POL}}$ represent the phase space density (PSD) of Bell et al. [2002] from POLAR in units of, e.g., $\mathrm{s}^{3} / \mathrm{cm}^{6}$, and let $f_{0}^{\mathrm{CRR}}$ represent the PSD of Bortnik et al. [2007a] from CRRES in the same units. Define the "weights" of the two distributions at a given $\mathrm{L}$ shell, $L_{\text {meas }}$, for a given plasmapause extent, $L_{\mathrm{PP}}$, as

$$
\begin{aligned}
w^{\mathrm{POL}} & =\frac{\exp \left(-\alpha\left(L_{\text {meas }}-L_{\mathrm{PP}}\right)\right)}{1+\exp \left(-\alpha\left(L_{\text {meas }}-L_{\mathrm{PP}}\right)\right)} \\
w^{\mathrm{CRR}} & =\frac{\exp \left(\alpha\left(L_{\text {meas }}-L_{\mathrm{PP}}\right)\right)}{1+\exp \left(\alpha\left(L_{\text {meas }}-L_{\mathrm{PP}}\right)\right)} .
\end{aligned}
$$

Then, the implemented hybrid PSD is given by the weighted mean in log-space of POLAR and CRRES PSDs as

$$
f_{0}^{\text {hybrid }}=\exp \left(\frac{\log \left(f_{0}^{\mathrm{POL}}\right) w^{\mathrm{POL}}+\log \left(f_{0}^{\mathrm{CRR}}\right) w^{\mathrm{CRR}}}{w^{\mathrm{POL}}+w^{\mathrm{CRR}}}\right) .
$$

[36] Reasonable results are obtained with $\alpha=5$. For reference, when $L_{\text {meas }}-L_{\mathrm{PP}}=0$, the two distributions are weighted equally in log-space, and when $L_{\text {meas }}-L_{\mathrm{PP}}=+(-)$ 0.5 , i.e., the measurement location is $0.5 \mathrm{~L}$ shells beyond (within) the plasmapause, $f_{0}^{\mathrm{CRR}}$ is weighted 12 times more (less) than $f_{0}^{\mathrm{POL}}$ in log-space.

[37] It should be noted that, although this ray tracing procedure is three dimensional, the following study is restricted to rays that lie approximately in a single meridional plane. Due to azimuthal gradients in the plasma and $B$ field models, rays exhibit a slight tendency to propagate to earlier local times with increasing $\mathrm{L}$ shell. The maximum azimuthal deviation of any ray considered in this study is $18^{\circ}$ (1.2 hours in MLT), with an average maximal deviation per ray of $7^{\circ}(0.5$ hours in MLT). Because this value is small, the local time deviation of rays is neglected in this study, and wave normals and positions are given in two dimensions with respect to the meridional plane of the rays.

\subsection{Ray Tracing Procedure}

[38] Rays are launched in the vicinity of Palmer, at $\lambda=$ $50^{\circ} \mathrm{S}, \mathrm{MLT}=06, \mathrm{UT}=10$. The GCPM and Tsyganenko models for plasma density and magnetic field are used, and the rays propagate in the nonducted mode. Rays are launched at $1000 \mathrm{~km}$ altitude, with 80 equally spaced magnetic latitudes within $1000 \mathrm{~km}$ of $50^{\circ} \mathrm{S}$, and with 13 equally spaced $\mathrm{k}$ vector angles directed away from the Earth within the transmission cone, for a total of 1040 rays per simulation.

[39] The transmission cone angle defines the maximum deviation of downward-directed $\mathrm{k}$ vectors, with respect to the normal to the Earth's surface, that may penetrate through the ionosphere and to the ground without suffering total internal reflection at the boundary between the lower edge of the ionosphere and free space [e.g., Helliwell, 1965, section 3.7]. To calculate the transmission cone, it is assumed that the plasma density from the ray origin to the ground may be approximated as a stratified medium, and therefore that the horizontal component of the $\mathrm{k}$ vector is conserved. At $1 \mathrm{kHz}$ and $4 \mathrm{kHz}$, two frequencies of interest for this study, the half angle of the transmission cone, measured from the vertical, is $0.84^{\circ}$ and $1.44^{\circ}$, respectively.

[40] Each ray is traced for up to $30 \mathrm{~s}$ or until it either impacts the Earth or departs from the precalculated density grid in the range $-4 \leq X_{\mathrm{SM}} \leq 4,-8 \leq Y_{\mathrm{SM}} \leq 0,-3 \leq Z_{\mathrm{SM}} \leq 3$, where all coordinates are in units of Earth radii in the solarmagnetic coordinate system. In practice, under these criteria, 
(a)
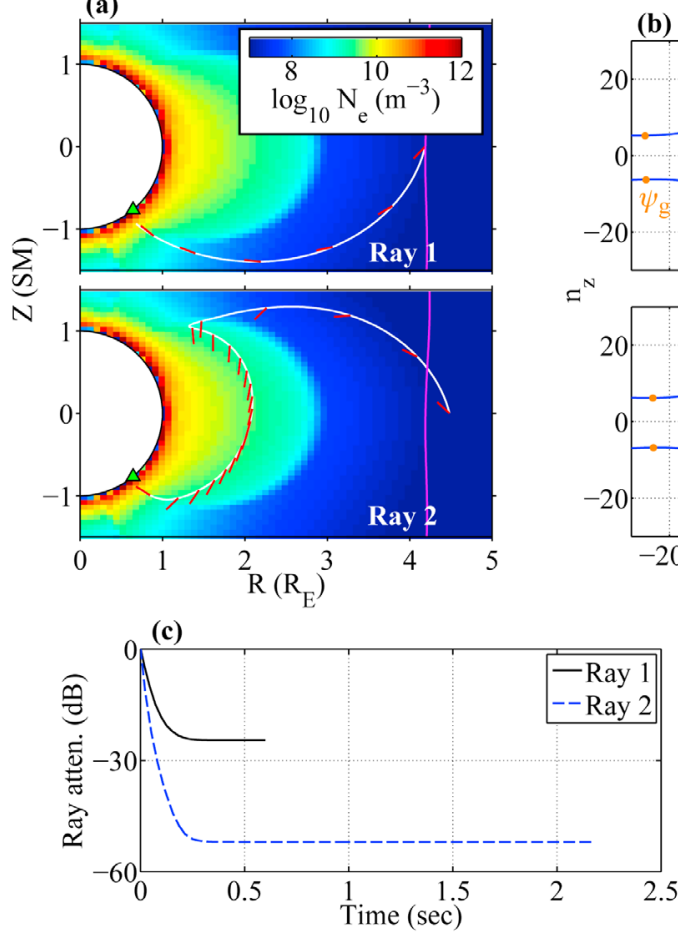

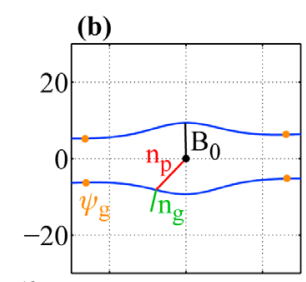

$z^{N}$

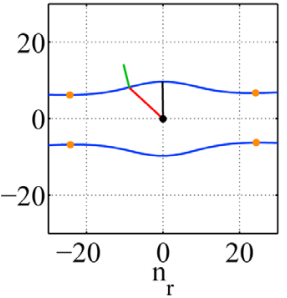

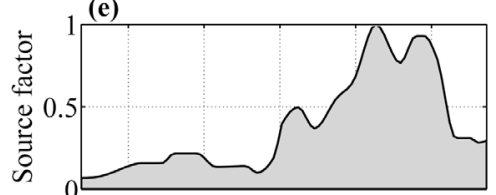
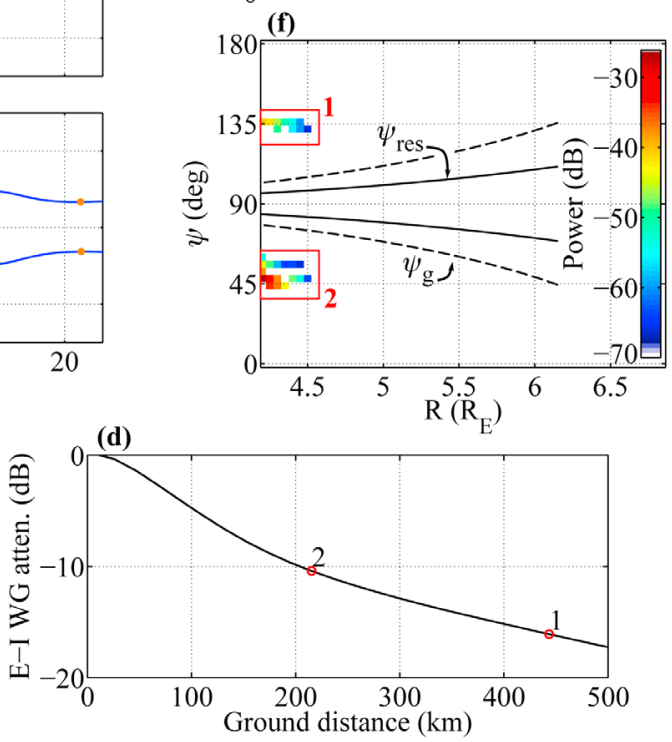

Figure 6. Two $1 \mathrm{kHz}$ ray families that are capable of being received at Palmer. (a) Representative raypaths from each of two ray families. Family 1 is the direct path from the source region to Palmer, and family 2 includes rays that magnetospherically reflect into the plasmasphere before their reception on the ground. (b) Initial refractive index surfaces for example rays. (c) Attenuation of example rays versus time over the course of ray tracing, via Landau damping. (d) Attenuation of example rays versus distance within the Earth-ionosphere waveguide, via full wave modeling. (e) Source factor showing relative expected chorus versus radial extent. (f) Source attenuation plot of relative received power versus wave normal $\psi$ and radial extent. Solid lines indicate the local resonance cone angle, $\psi_{\text {res}}$, and dashed lines indicate the local Gendrin angle, $\psi_{g}$. The two families of similar rays, labeled 1 and 2 , correspond to the two example rays from the previous panels.

no rays survive beyond $10 \mathrm{~s}$. Each time a ray crosses the equatorial plane, the local plasma density and gyrofrequency are examined. If the ray is (1) outside the plasmasphere and (2) within the range $0.1 f_{c e q} \leq f \leq 0.5 f_{c e q}$ (where $f_{c e q}$ is the equatorial electron gyrofrequency along the given field line), which is the frequency range of lower band chorus [Tsurutani and Smith, 1974; Burtis and Helliwell, 1976], then that point is saved as a potential chorus source location. A single original ray may give rise to more than one potential chorus source location if it exhibits multiple magnetospheric reflections.

[41] The chorus source region (i.e., the region from which chorus is truly generated, which is not the same as the location from which the "reverse" rays are launched) is considered to lie on the equatorial plane, with initial wave normal angles uniformly distributed within the resonance cone. Although several satellite studies have attempted to characterize the wave normal distribution of the equatorial chorus source [e.g., Haque et al., 2010, and references therein], statistics have generally been too low to draw any definitive conclusions, leading to our use of a uniform distribution in this study. The source region is binned on two parameters: $R$, the distance from the center of the Earth in the equatorial plane, and $\psi$, the initial wave normal angle with respect to the ambient magnetic field. Each bin is of uniform size, with $\Delta R=0.05 R_{\mathrm{E}}$ and $\Delta \psi=4^{\circ}$.

[42] Chorus rays that can reach Palmer tend to occur in several distinct "families," or groupings of rays with similar initial wave normals and radial extent. Figure 6 shows several facets of the ray tracing procedure, along with example rays from the two ray families that are present at $1 \mathrm{kHz}$. For this simulation, $L_{\mathrm{PP}}=2.9$. The ray tracing procedure is described below with reference to Figure 6 .

[43] Figure 6a shows representative rays from the two ray families. We interpret the rays in their "forward" sense, as if they were originally launched from the equatorial plane and eventually arrived at $1000 \mathrm{~km}$ altitude. Ray paths are shown in white, with wave normals shown as red ticks, equally spaced every $100 \mathrm{~ms}$. The magenta line indicates a contour of $f / f_{\text {ceq }}=0.1$; all chorus generation happens at values of $R$ beyond this boundary. The upper bound on $f_{c e q}$ for chorus generation, at $f / f_{\text {ceq }}=0.5$ is beyond the scale of the image, at $R \sim 7 \mathrm{R}_{\mathrm{E}}$. Palmer's location is indicated by the green triangle at $\lambda=-50^{\circ}$ on the surface of the Earth. The background image is a meridional slice of the GCPM electron density. Ray family 1 consists of rays that propagate directly from the chorus source region to Palmer without magnetospherically reflecting (MR), and family 2 consists of rays that MR at the plasmapause boundary, which allows them 
access into the plasmapause before reaching Palmer. Because ray tracing is performed in three dimensions, the ray paths and wave normals have been projected into the $\mathrm{MLT}=06$ meridional plane.

[44] Figure 6b shows the initial refractive index surfaces for the representative rays. The direction of the ambient magnetic field, $B_{0}$, the wave refractive index, $n_{p}=c / v_{p}$, and the group refractive index, $n_{g}=c / v_{g}$, as well as the Gendrin angle, $\psi_{g}$, are indicated, where $c$ is the speed of light in free space, $v_{p}$ is the wave phase velocity and $v_{g}$ is the wave group velocity. $n_{p}$ and $n_{g}$ point in the direction of the wave $\mathrm{k}$ vector and group velocity vector, respectively.

[45] Each potential chorus source location represents a ray that originally begins with unity power and is attenuated in two separate steps. First, Figure $6 \mathrm{c}$ shows the attenuation of the representative rays over the course of their magnetospheric propagation due to Landau damping, as discussed in section 4.1. The majority of damping occurs at high $\mathrm{L}$ shells outside the plasmasphere. In particular, once ray 2 enters the plasmasphere, the attenuation due to Landau damping is negligible. Unlike some other studies of ray tracing [e.g., Bortnik et al., 2007a, 2007b], this study does not include a geometric effect in determining the power gain or loss due to the focusing of magnetic field lines at low altitudes. Instead, this focusing or defocusing happens naturally through the use of a large number of rays.

[46] The second mode of attenuation, shown in Figure 6d, is attenuation from Earth-ionosphere waveguide propagation. Each ray begins at $1000 \mathrm{~km}$ altitude with the injection point footprint a distance $d$ from Palmer Station, where $d \leq$ $1000 \mathrm{~km}$. Earth-ionosphere waveguide attenuation is calculated using the full wave model of Lehtinen and Inan [2008, 2009]. A summer nighttime ionospheric profile and a perfectly conducting ground layer (representative of Palmer's primarily all-sea paths) are used. A Gaussian wave packet of the appropriate frequency is injected at $140 \mathrm{~km}$ altitude with vertical (downward) wave normal. The ground power at various distances from the source is recorded, normalized by the ground power directly beneath the source. The resulting quantity $A(d)$ represents an attenuation factor for Earth-ionosphere waveguide propagation, as a function of $d$, by which each ray's power is multiplied. The full wave model is run only once for any given frequency, and the quantity $A(d)$ is assumed to be valid for all modeled rays within $1000 \mathrm{~km}$ of Palmer. The two example rays reach the ground at $\sim 450 \mathrm{~km}$ and $\sim 215 \mathrm{~km}$ from Palmer, respectively, and are marked as such in Figure $6 \mathrm{~d}$. When both Landau damping and Earth-ionosphere waveguide attenuation are considered, there can be wide variations in the attenuation of different rays in a given family, due to the fact that slight variations in initial conditions may give rise to large variations in propagation paths and ionospheric penetration points.

[47] Figure 6e is a plot of "source factor" as a function of radial extent, $R$. This plot is derived from work by Burtis and Helliwell [1976, Figure 9c], which shows chorus occurrence as a function of $f / f_{\text {ceq }}$. We define source factor as the observed occurrence of Burtis and Helliwell [1976, Figure 9c], normalized so that the maximum value is 1 . Here, source factor is plotted against $R$, using the T96 magnetic field model to map from $f / f_{c e q}$ to $R$. The source factor plot is then the relative expected likelihood of observing a $1 \mathrm{kHz}$ chorus source at a given radial extent in the equatorial plane. Because the measurements of Burtis and Helliwell [1976] include both waves inside and outside the plasmasphere, it is possible that the observed chorus percentage is artificially low at low $f / f_{c e q}$ or $R$ due to those measurements being taken within the plasmasphere where chorus is generally not observed. The use of the source factor in deriving the Chorus Availability Factor (CHAF) is discussed in section 4.3, and due to the possible confounding effects of its constituent data containing measurements inside the plasmasphere, CHAF is derived both with and without implementing the source factor.

[48] After building a list of potential chorus source locations from the 1040 original rays, the amplitude of any given $R-\psi$ bin is set to the maximum ray amplitude in that bin after attenuation both via Landau damping in the magnetosphere and via attenuation in the Earth-ionosphere waveguide. We refer to a plot of the binned results for a simulation with a given wave frequency and plasmapause extent as a "source attenuation plot."

[49] Figure 6f shows a source attenuation plot for a simulation where $L_{\mathrm{PP}}=2.9$, from which the two example rays are drawn. The local resonance cone angle, $\psi_{\text {res }}$, defined as the wave normal angle at which the magnitude of the refractive index goes to infinity, is indicated by the solid black lines. The local Gendrin angle, $\psi_{g}$, defined as the nonzero wave normal angle at which the group velocity vector is parallel to the static magnetic field, is indicated by the dashed black lines. The two separate ray families, from which the above example rays are drawn, are highlighted with red boxes. The rays do not show any particular relationship with the resonance cone or Gendrin angles.

[50] Figure 7 is analogous to Figure 6 , but for $4 \mathrm{kHz}$ waves. Because $f$ is increased, the magenta lines, indicating the contours of $f l f_{c e q}=0.1$ and $f l f_{c e q}=0.5$ are now closer to the Earth, and both boundaries of the chorus source region can be seen. In addition, there are now four ray families, representing the direct path, and one, two and three magnetospheric reflections. In all cases, the damping is most significant at large $\mathrm{L}$ shells outside the plasmasphere, where wave normals are most oblique. Rays 3 and 4 begin with their wave normals directed away from the Earth, near the resonance cone. After the first magnetospheric reflection, they appear to be guided by the plasmapause boundary before reflecting from the inner boundary. This has the effect of rotating the wave normal toward the Earth, allowing the rays to reach the ground. Because Rays 3 and 4 spend more time outside the plasmasphere, and have more highly oblique wave normals than do rays 1 and 2, they are damped more heavily during their propagation.

[51] In the $4 \mathrm{kHz}$ case, the initial wave normals of some ray families do show a relationship with the resonance cone and Gendrin angles. Some rays from families 1 and 2 tend to be generated near the Gendrin angle, while some rays from families 3 and 4 tend to be generated near the resonance cone angle. The associations are loose, and no ray families appear constrained to either the resonance cone or the Gendrin angle. The relation between the wave normals of ray family 1 (the direct path) and the Gendrin angle is consistent with the work of Chum and Santolik [2005], who found that certain rays generated with wave normals in the vicinity of the Gendrin angle would reach low altitudes and 
(a)

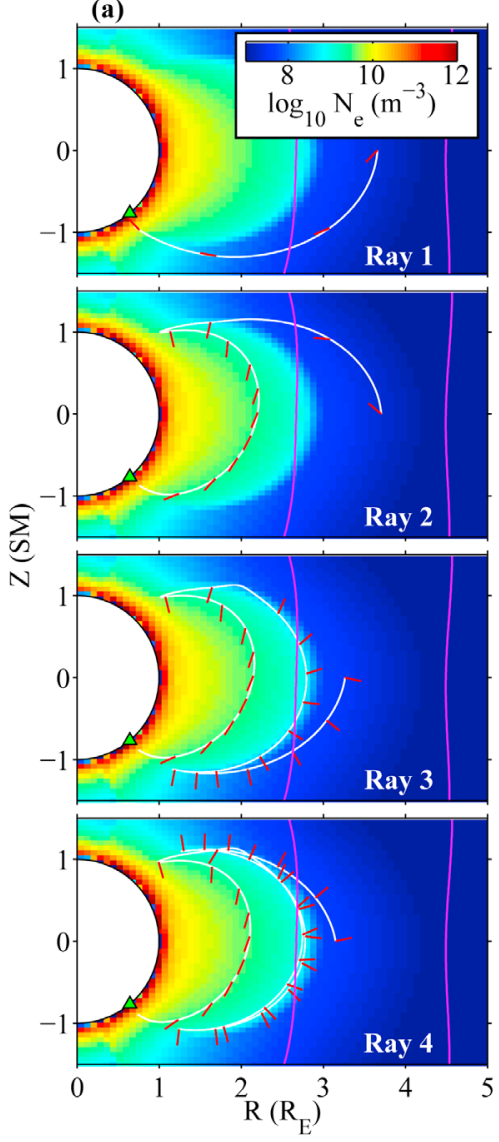

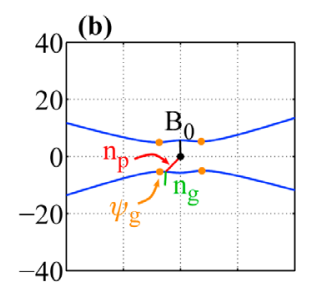
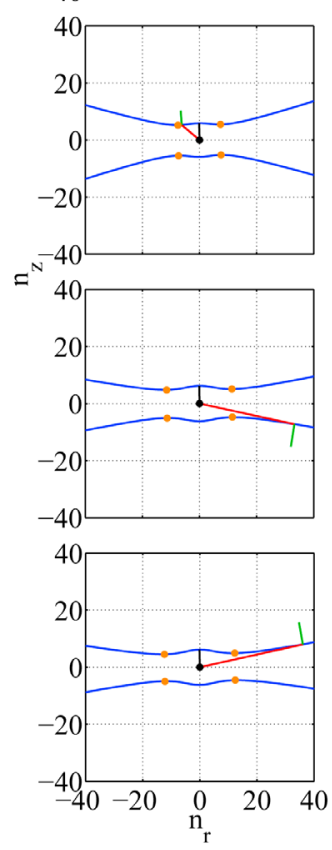
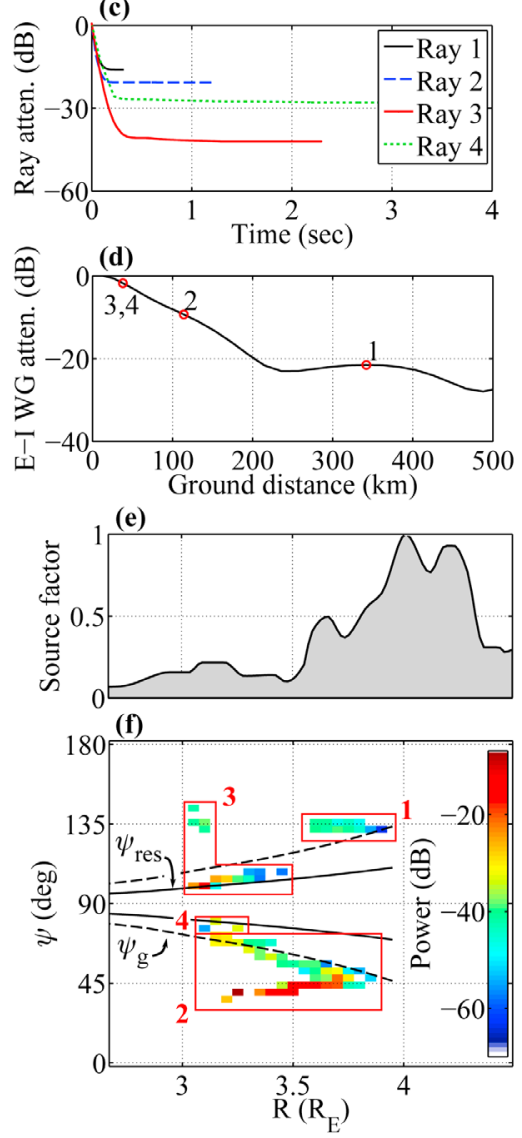

Figure 7. Same as Figure 6 but for $4 \mathrm{kHz}$. At this frequency, there are four distinct ray families, representing the direct path and one, two, and three magnetic reflections.

possibly penetrate to the ground before being magnetospherically reflected. Although this behavior is seen in our results at $4 \mathrm{kHz}$, it is not observed at $1 \mathrm{kHz}$. This is possibly due to the fact that Chum and Santolik [2005] did not include Landau damping in their calculations. Although some $1 \mathrm{kHz}$ rays in our study do begin at the equatorial plane with wave normals near the Gendrin angle, those waves are damped to negligible power in the simulation, and therefore do not appear on the source attenuation plot in Figure 6f.

\subsection{Chorus Availability Factor}

[52] Figures $6 \mathrm{f}$ and $7 \mathrm{f}$ showed source attenuation plots at $1 \mathrm{kHz}$ and $4 \mathrm{kHz}$ for a single plasmapause extent, $L_{\mathrm{PP}}=$ 2.9. This analysis is repeated for many different values of $L_{\mathrm{PP}}$ to gain insight into the particular way in which the plasmapause extent affects the ability for chorus waves to propagate from their source to Palmer. Figure 8 shows source attenuation plots for $1 \mathrm{kHz}$ (upper panels) and $4 \mathrm{kHz}$ (lower panels) for plasmapause extents in the range $2.1 \leq L_{\mathrm{PP}} \leq 4.3$. The color scale has been changed slightly for clarity.

[53] Initially, we focus our discussion on the $1 \mathrm{kHz}$ case, in the upper panels of Figure 8. At the greatest plasmapause extent, $L_{\mathrm{PP}}=4.3$, rays from the chorus source region are not accessible to Palmer; reverse rays launched from Palmer are either unable to escape the plasmasphere, and instead reflect off of its inner boundary before impacting the ionosphere in the conjugate hemisphere, or they escape the plasmasphere with oblique wave normals and are heavily damped before crossing the equatorial plane. As the plasmasphere becomes more eroded down to $L_{\mathrm{PP}}=2.9$, although rays as far out as $L=7$ are accessible to Palmer (not shown), most are severely damped; only certain rays that originate within $4.2 \lesssim L \lesssim 4.6$ sufficiently avoid damping to be received above the $-70 \mathrm{~dB}$ cutoff. Erosion of the plasmasphere beyond $L_{\mathrm{PP}}=2.9$ results in increased propagation time outside the plasmasphere, and hence, increased damping, particularly for waves with initial wave normals $\psi \sim 50^{\circ}$. The situation is similar for $4 \mathrm{kHz}$. For high $L_{\mathrm{PP}}$, rays from the chorus source region cannot reach Palmer; reverse rays are unable to escape the plasmasphere. For $L_{\mathrm{PP}} \sim 2.9$, a maximum of rays reach Palmer with significant power. For low $L_{\mathrm{PP}}$, as for high $L_{\mathrm{PP}}$ most reverse rays launched from Palmer do not escape the plasmasphere.

[54] One important difference between the simulations at $1 \mathrm{kHz}$ and $4 \mathrm{kHz}$ is where the plasmapause lies with respect to the extents of the chorus source region, defined by $0.1 \leq$ $f / f_{\text {ceq }} \leq 0.5$. At $1 \mathrm{kHz}$, the source region is in the range $4.2 \leq L \leq 6.9$, which is beyond the plasmapause for almost all simulations. However, at $4 \mathrm{kHz}$, the source region is in the range $2.7 \leq L \leq 4.5$, which means that for many of the simulations, the plasmasphere overlaps the 

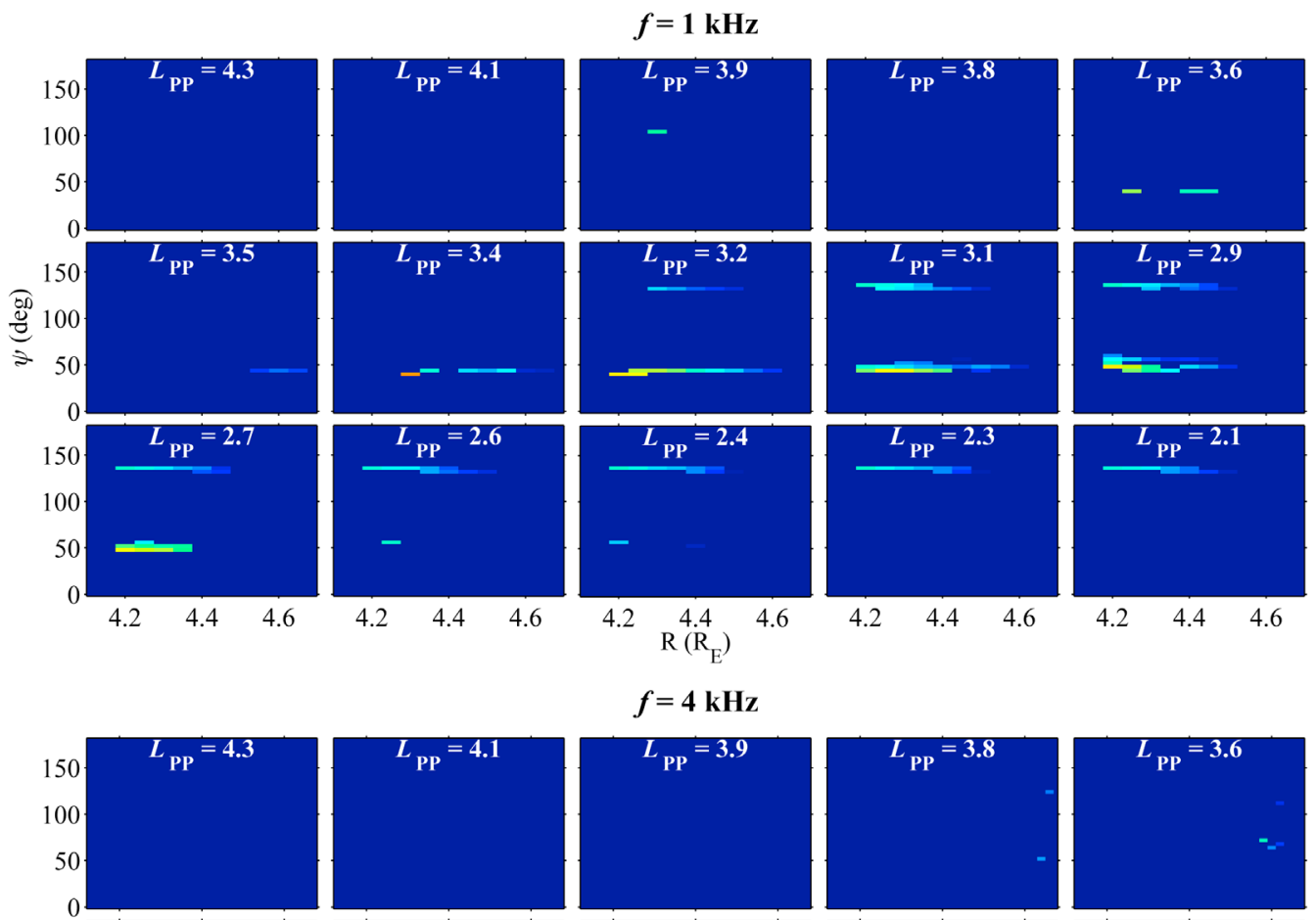

\section{$f=4 \mathrm{kHz}$}
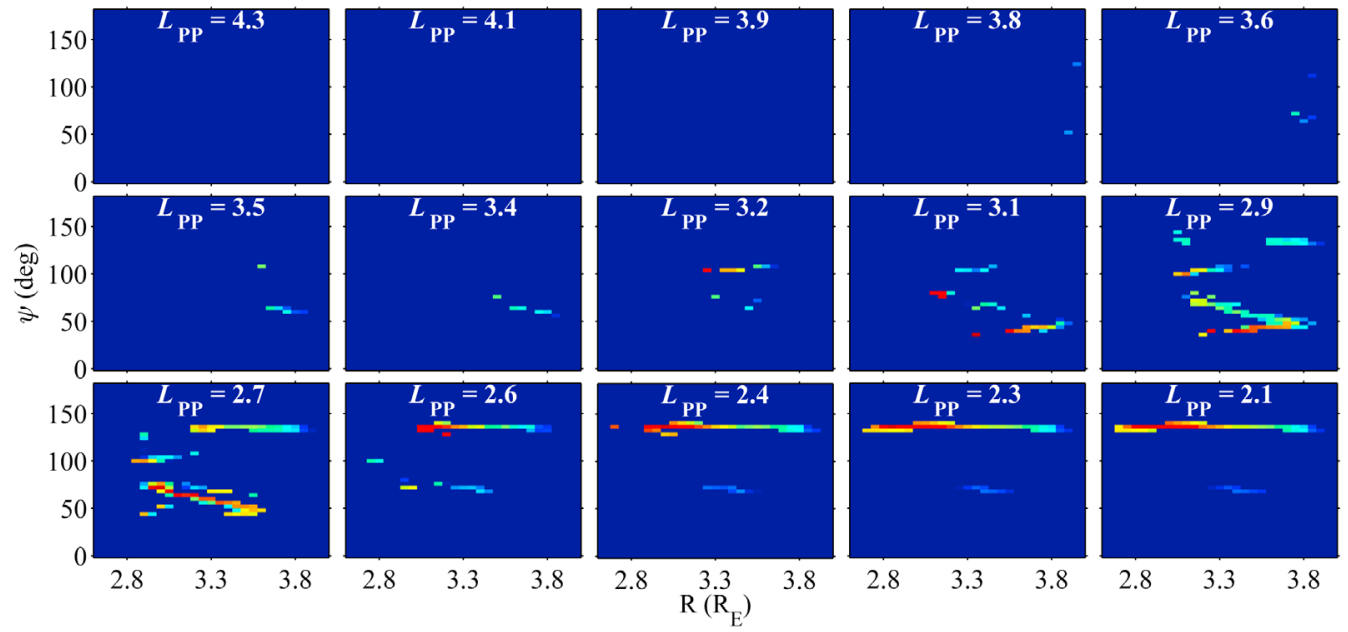

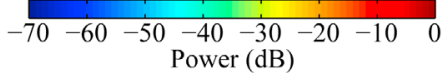

Figure 8. Source attenuation plots for (upper panels) $f=1 \mathrm{kHz}$ and (lower panels) $f=4 \mathrm{kHz}$ for plasmapause extents in the range $2.1 \leq L_{\mathrm{PP}} \leq 4.3$. Note that the scales of the $x$ axes in the upper and lower panels are not the same.

chorus source region. This is why, in the lower panels of Figure 8, the chorus source region appears to expand to the left as $L_{\mathrm{PP}}$ decreases. The plasmapause is moving to the left of the plots, and a greater portion of the chorus source region is becoming available.

[55] Because rays may be substantially damped over the course of propagation, in order to properly analyze the results of the simulations, it is necessary to define a "minimum detectable ray power," below which rays are excluded from the analysis. To first order, this can be achieved by comparing the mean power observed on the ground with the mean power observed via in situ measurements. A histogram of observed amplitudes over the course of this study, overlaid with the associated probability distribution, is shown in Figure 9. Chorus amplitudes observed at Palmer are distributed approximately lognormally, as

$$
A_{\mathrm{dB}} \sim \ln \mathcal{N}\left(\mu=3.5, \sigma^{2}=0.036\right),
$$

with mean $35 \mathrm{~dB}-\mathrm{fT}$ and standard deviation $6.8 \mathrm{~dB}-\mathrm{fT}$. The observed mean amplitude of $35 \mathrm{~dB}$-fT at Palmer can be compared with the mean $B$ field amplitude calculated by Santolik [2008], based on equatorial chorus $E$ field measurements from Meredith et al. [2001], of 10-100 pT, or 80$100 \mathrm{~dB}-\mathrm{fT}$. Comparing the two numbers, up to $\sim 65 \mathrm{~dB}$ of attenuation is expected from the equatorial source region to Palmer. However, in this analysis, we are not modeling attenuation suffered through transionospheric propagation. Transionospheric attenuation is expected to be on the order of $\sim 5 \mathrm{~dB}$, somewhere between the daytime and nighttime attenuation calculations of Helliwell [1965, Figure 3-35], for $2 \mathrm{kHz}$ waves (since our simulations are run at $06 \mathrm{MLT}$ ). This leaves an expected attenuation from Landau damping and Earth-ionosphere waveguide losses of $\sim 60 \mathrm{~dB}$. To account for the lower end of our observed power distribution, which reaches down to $\sim 25 \mathrm{~dB}-\mathrm{fT}$ in Figure 9, an additional $10 \mathrm{~dB}$ of loss is allowed. Thus, we define our minimum detectable 


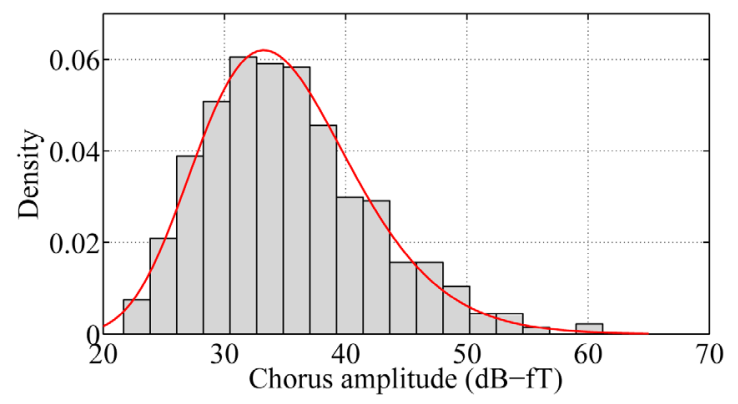

Figure 9. Histogram and lognormal probability distribution function (PDF) of observed chorus amplitudes at Palmer. Bins of the histogram have been normalized by the total number of samples and the bin width so that they have the same units as the lognormal PDF.

ray power to be $-70 \mathrm{~dB}$. Although it is necessary to define a minimum detectable ray power to perform the following analysis, our conclusions are not strongly dependent on its exact value.

[56] We define the CHAF for a given frequency and $L_{\mathrm{PP}}$ as follows. First, all bins of a given simulation (i.e., from a source attenuation plot, such as Figure $6 \mathrm{f}$ ) are normalized by adding $70 \mathrm{~dB}$ to each bin, which ensures that bin values are all positive, between 0 and $70 \mathrm{~dB}$. Then, each bin is multiplied by the source factor (e.g., Figure 6e), at its particular radial extent. For example, the bins at the lowest radial extent in Figure $6 \mathrm{f}$, at $R \sim 4.2 \mathrm{R}_{\mathrm{E}}$, are multiplied by the source factor of Figure 6e at that same radial extent, which is approximately 0.05 . This has the effect of reducing the influence of bins that are at radial extents at which chorus is less commonly observed. Finally, the values of the bins are summed, and the resulting scalar quantity, as a function of frequency and $L_{\mathrm{PP}}$, is the CHAF.

[57] The CHAF of the $1 \mathrm{kHz}$ and $4 \mathrm{kHz}$ simulations is shown in the left panels of Figure 10. The calculated CHAF both before and after applying the source factor are shown in gray and black lines, respectively. It can be seen that application of the source factor makes only a minor difference in the trend of CHAF with $L_{\mathrm{PP}}$ for either frequency. This shows that, even if the fact that chorus is preferentially generated at certain values of $f \mid f_{\text {ceq }}$ is not included (for example, due to the fact that the source factor may artificially reduce the effect of chorus originating at low L shells outside the plasmasphere) the plasmapause has a similar effect in dictating the amplitude of received chorus.

[58] Because CHAF is derived from the data that makes up the source attenuation plots in Figure 8, its behavior with respect to $L_{\mathrm{PP}}$ is analogous to that in Figure 8. As $L_{\mathrm{PP}}$ decreases from $L_{\mathrm{PP}}=4.3$ to $L_{\mathrm{PP}}=2.1$, the availability of different portions of the chorus source region wax and wane, which translates into increasing and decreasing CHAF. At $1 \mathrm{kHz}, \mathrm{CHAF}$ is maximized for $L_{\mathrm{PP}}=3.1$, where two regions, narrow in $\psi$ and broad in $R$ are accessible to Palmer. At $4 \mathrm{kHz}$, CHAF is maximized for $L_{\mathrm{PP}}=2.9$, where several broad regions of the source region are accessible to Palmer. These regions are made up of rays from the different ray families discussed in section 4.2.

\subsection{Comparison With Observations}

[59] We would like to compare the simulated CHAF to the experimental results of section 3.2. If the variation in chorus occurrence as a function of $L_{\mathrm{PP}}$ observed in section 3.2 is primarily a propagation effect, then CHAF should behave similarly to the empirically modeled normalized chorus occurrence probability, $\mu$, as a function of $L_{\mathrm{PP}}$. Note that CHAF is merely a proxy measurement of chorus observed probability and is not a probability. To form a proper probability estimation from this data, it would be necessary to estimate the distribution of chorus power as a function of radial distance or $f / f_{c e q}$ and initial wave normal angle. For

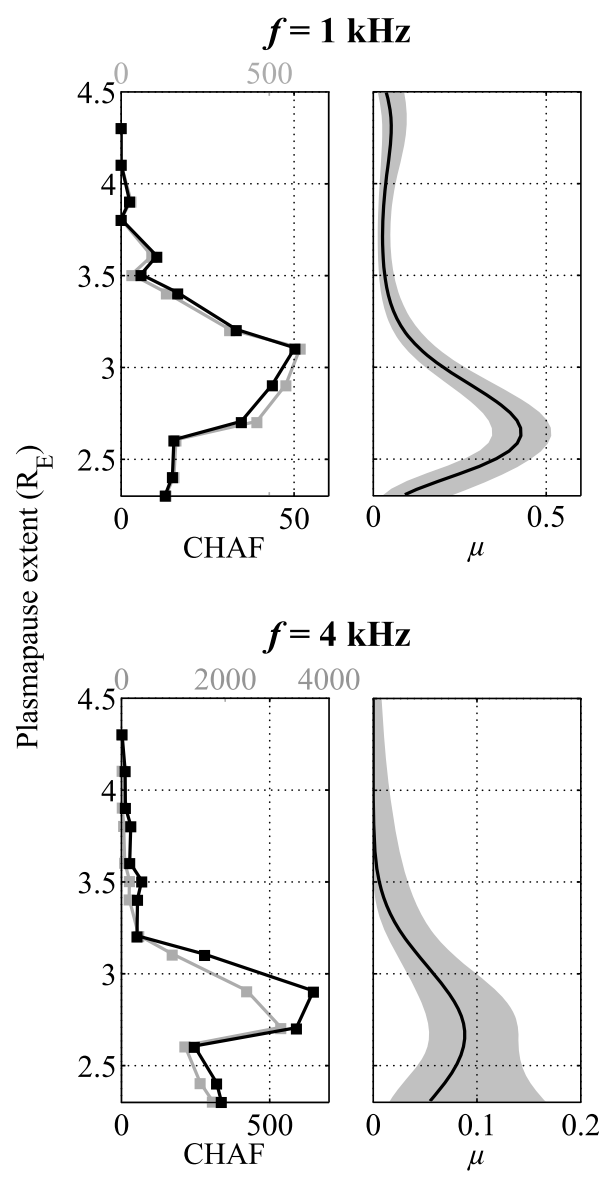

Figure 10. Comparison of (left) CHAF from ray tracing with (right) generalized linear model of occurrence probability, $\mu$, derived from measurements. CHAF is calculated from constituent data of the source attenuation plots of Figure 8 , and $\mu$ is calculated using data at single frequencies 1 and $4 \mathrm{kHz}$, instead of a range of frequencies as in section 3.2. As before, on the plot of $\mu$, the solid black line indicates $\mu$, and the shaded area indicates the $95 \%$ confidence interval. The lighter-colored lines on the plots of CHAF represent the values prior to applying the source factor from Burtis and Helliwell [1976], and the darker lines represent values after applying the source factor. The shapes of the lines with and without the source factor applied are very similar. Note that the scales of the $x$ axes in the upper and lower panels, and on the overlaid plots of CHAF, are not the same. 
lack of this information, we have assumed uniform initial power at all wave normals and radial distances.

[60] Figure 10 shows a comparison of the CHAF at 1 and $4 \mathrm{kHz}$ with the equivalent univariate generalized linear model (GLM) results for $\mu$. The GLM results shown here are limited to chorus occurring at 1 and $4 \mathrm{kHz}$, instead of the ranges $f<1.5 \mathrm{kHz}$ and $f>3 \mathrm{kHz}$ shown in Figure 4. First, and most importantly, the saturation effect is reproduced for both frequencies. Both $\mu$ and CHAF initially increase with decreasing $L_{\mathrm{PP}}$, reach a peak, and then decrease. Their peaks are within $0.5 \mathrm{~L}$. This similarity between $\mathrm{CHAF}$ and $\mu$ is strongly indicative of the fact that the behavior of $\mu$ with respect to $L_{\mathrm{PP}}$ is a propagation effect and not a source effect (since only propagation effects are included in the ray tracing).

[61] However, we also note the important discrepancy between the $L_{\mathrm{PP}}$ values for the peaks of CHAF and the peaks of $\mu$. For $1 \mathrm{kHz}$, the peak of $\mu$ is at $L_{\mathrm{PP}}=2.6$, whereas that for CHAF is at $L_{\mathrm{PP}}=3.1$, a difference of $0.5 \mathrm{R}_{\mathrm{E}}$. The random error in the measured value of $L_{\mathrm{PP}}$ for either $\mu$ (measured by clicking on equatorially mapped EUV images) or CHAF (measured by direct examination of an equatorial slice through the GCPM grid) is estimated to be $\pm 0.1 \mathrm{R}_{\mathrm{E}}$, but this is too small to account for the observed discrepancy. Similarly for $4 \mathrm{kHz}$, the observed peaks are at $L_{\mathrm{PP}}=2.7$ and $L_{\mathrm{PP}}=2.9$, respectively, a smaller difference of $0.2 \mathrm{R}_{\mathrm{E}}$.

[62] There are several different possible causes for the discrepancy between the peaks in $\mu$ and CHAF. The first and most obvious cause may be errors in particle densities from the GCPM density model, either in the absolute density or in density gradients. The GCPM model necessarily represents "averaged" conditions for its input values, and may contain systematic biases with respect to the true magnetospheric conditions under which chorus is observed at Palmer.

[63] Another cause may lie in our use of a hybrid energetic electron distribution when calculating Landau damping. The CRRES distribution used outside the plasmasphere uses data from disturbed periods, when AE $>300 \mathrm{nT}$. However, the POLAR distribution used inside the plasmasphere uses data from quiet-to-moderate conditions, when $\mathrm{K}_{\mathrm{p}} \leq 4$. Because chorus tends to peak during active periods, the use of quiet/moderate fluxes within the plasmasphere has the effect of artificially lowering the energetic particle flux inside the plasmasphere, therefore lowering the damping coefficients and allowing rays to propagate for a long time within the plasmasphere. Thus, at $1 \mathrm{kHz}$, ray family 2 from Figure 6, which involves extended propagation within the plasmasphere, and which is dominant for $L_{\mathrm{PP}} \gtrsim 2.7$, may be less influential than modeled.

[64] Finally, by excluding the prevalent density irregularities that permeate the plasmasphere [e.g., Carpenter et al., 2002, and references therein], we neglect what may be a significant population of waves that are guided by these irregularities. In particular, in the real plasmasphere, density irregularities in the vicinity of the plasmapause may preferentially guide waves to Palmer when the plasmapause is at lower L shells [Inan and Bell, 1977]. The exclusion of irregularities is an inevitable consequence of using an "averaged" plasma density model, such as the GCPM model for the plasma density. A full discussion of the effects of guiding by density irregularities is beyond the scope of this study.

[65] One other important discrepancy between the plots of $\mu$ and CHAF is that the relative value of $\mu$ for low frequencies is significantly greater than that for high frequencies (right panels), whereas the opposite relation is true for CHAF (left panels). This may be due to the fact that higher-frequency waves tend to be generated with lower amplitudes [Burtis and Helliwell, 1975], whereas we have assumed in our ray tracing analysis that the amplitude of generated waves is the same across all frequencies.

\section{Conclusions}

[66] We have proposed in this study that the extent of the plasmapause, denoted $L_{\mathrm{PP}}$, plays a large role in determining the ability for chorus waves to propagate from their equatorial magnetospheric source region to the ground. Using wave data from the ground-based receiver at Palmer Station, Antarctica, together with plasmapause data from the IMAGE EUV instrument, a generalized linear model regression was employed in section 3.2 to show the strong dependence of chorus normalized occurrence on $L_{\mathrm{PP}}$.

[67] The separability of $\mathrm{AE}$ and $L_{\mathrm{PP}}$ shown in section 3.3 provides evidence that the dependence of chorus occurrence on $L_{\mathrm{PP}}$ is in fact a propagation effect, and not simply a confounding source effect (i.e., a consequence of the fact that magnetic substorms both give rise to chorus generation and, separately, cause erosion of the plasmasphere). In particular, Figure 5 shows that the general trend of normalized occurrence versus plasmapause persists across a wide range of $\mathrm{AE}$ values. This shows that the relation of chorus occurrence to AE (a proxy measure of a source effect), is separable from the relation of chorus occurrence to $L_{\mathrm{PP}}$ (a measure of a propagation effect), and therefore, that there is a significant influence of instantaneous plasmapause extent in determining whether chorus can reach Palmer.

[68] These conclusions were solidified via a reverse ray tracing study. By launching rays from Palmer and tracking their power, wave normal, and equatorial crossings through the expected chorus source region, a measure of the portion of the chorus source region from which rays may reach Palmer was obtained, which we termed the Chorus Availability Factor, or CHAF. The most salient similarity between how the experimentally observed chorus occurrence $(\mu)$ and the ray tracing model (CHAF) depend on $L_{\mathrm{PP}}$ is the so-called saturation effect, where during experimental observations, chorus is observed on the ground most often for $L \sim 2.6$. It was shown in section 4.4 that this effect is reproduced via ray tracing (with a small systematic error in the exact value of $L_{\mathrm{PP}}$ ) by varying only $L_{\mathrm{PP}}$; this eliminates the possibility of a confounding source effect, and further enforces the conclusion that the plasmapause extent has a direct effect on allowing chorus access to the ground.

[69] The peak of the saturation, either the observed peak of $2.6 \lesssim L_{\mathrm{PP}} \lesssim 2.7$ or the modeled peak of $2.9 \lesssim L_{\mathrm{PP}} \lesssim 3.1$, is somewhat higher than Palmer's location at $L=2.4$. One might naïvely expect the peak of chorus to occur at $L_{\mathrm{PP}}=$ 2.4 , because it is at that plasmasphere extent that Palmer Station lies on the plasmapause boundary. However, this theory neglects the mechanism of rays reaching Palmer via magnetospherically reflecting at the plasmapause boundary, 
as in ray family 2 from Figure 6 and ray families 2, 3, and 4 from Figure 7. This can occur at high plasmapause extents, and the prevalence of this mode of propagation may be one explanation for why chorus is often observed at Palmer even when the plasmapause is beyond $L=2.4$.

[70] Additionally, by ray tracing in a smooth magnetosphere (except for the obvious density gradient of the plasmapause itself), it was shown that it is possible for chorus to reach the ionosphere within the transmission cone and penetrate to the ground in the absence of any fieldaligned guiding structures. This is in contrast to long-held colloquial belief that only ducted chorus may access the ground. In fact, in light of the similarities between the ray tracing and the experimentally observed results, it seems plausible that nonducted chorus is the dominant mode of chorus observed on the ground. Without the constraint of a field-aligned guiding structure, chorus is able to cross L shells as it propagates from the source region to the ground. This explains why Palmer Station, located at a significantly lower L shell than that of the typical chorus source region, is able to observe chorus as often as it does.

[71] We conclude by saying that, due to the fact that midlatitude ground observations of chorus are likely to result from nonducted propagation, these observations are by no means limited to chorus source regions that lie on the same L shell as the receiver. In addition, plasmapause extent is an often neglected but critically important factor in determining chorus propagation to low altitudes and the ground.

[72] Acknowledgments. The work at Stanford University was supported by the National Science Foundation under award 0538627 and the Office of Naval Research under awards N00014-09-1-0034 and Z882802.

[73] Robert Lysak thanks Yuri Shprits and another reviewer for their assistance in evaluating this paper.

\section{References}

Abel, B., and R. M. Thorne (1998), Electron scattering loss in Earth's inner magnetosphere: 1. Dominant physical processes, J. Geophys. Res., 103 2385-2396, doi:10.1029/97JA02919.

Bell, T. F., U. S. Inan, J. Bortnik, and J. D. Scudder (2002), The Landau damping of magnetospherically reflected whistlers within the plasmasphere, Geophys. Res. Lett., 29(15), 1733, doi:10.1029/2002GL014752.

Bortnik, J., R. M. Thorne, and N. P. Meredith (2007a), Modeling the propagation characteristics of chorus using CRRES suprathermal electron fluxes, J. Geophys. Res., 112, A08204, doi:10.1029/2006JA012237.

Bortnik, J., R. M. Thorne, N. P. Meredith, and O. Santolík (2007b), Ray tracing of penetrating chorus and its implications for the radiation belts, Geophys. Res. Lett., 34, L15109, doi:10.1029/2007GL030040.

Bortnik, J., R. M. Thorne, and N. P. Meredith (2008), The unexpected origin of plasmaspheric hiss from discrete chorus emissions, Nature, 452, 62-66, doi:10.1038/nature06741.

Brinca, A. L. (1972), On the stability of obliquely propagating whistlers, J. Geophys. Res., 77, 3495-3507, doi:10.1029/JA077i019p03495.

Burch, J. L. (2000), IMAGE mission overview, Space Sci. Rev., 91, 1-14.

Burtis, W. J., and R. A. Helliwell (1975), Magnetospheric chorus: Amplitude and growth rate, J. Geophys. Res., 80, 3265-3270.

Burtis, W. J., and R. A. Helliwell (1976), Magnetospheric chorus: Occurrence patterns and normalized frequency, Planet. Space Sci., 24, 1007-1007, doi:10.1016/0032-0633(76)90119-7.

Carpenter, D. L. (1966), Whistler studies of the plasmapause in the magnetosphere: 1. Temporal variations in the position of the knee and some evidence on plasma motions near the knee, J. Geophys. Res., 71, 693-709.

Carpenter, D. L., and R. R. Anderson (1992), An ISEE/Whistler model of equatorial electron density in the magnetosphere, J. Geophys. Res., 97, 1097-1108, doi:10.1029/91JA01548.
Carpenter, D. L., and J. Lemaire (2004), The plasmasphere boundary layer, Ann. Geophys., 22, 4291-4298.

Carpenter, D. L., and D. M. Sulic (1988), Ducted whistler propagation outside the plasmapause, J. Geophys. Res., 93, 9731-9742, doi:10.1029/ JA093iA09p09731.

Carpenter, D. L., J. C. Foster, T. J. Rosenberg, and L. J. Lanzerotti (1975), A subauroral and midlatitude view of substorm activity, J. Geophys. Res., 80, 4279-4286.

Carpenter, D. L., et al. (2002), Small-scale field-aligned plasmaspheric density structures inferred from the Radio Plasma Imager on IMAGE, J. Geophys. Res., 107(A9), 1258, doi:10.1029/2001JA009199.

Chatterjee, S., and A. S. Hadi (2006), Regression Analysis by Example, 4th ed., Wiley-Interscience, Hoboken, N. J.

Chen, L., J. Bortnik, R. M. Thorne, R. B. Horne, and V. K. Jordanova (2009), Three-dimensional ray tracing of VLF waves in a magnetospheric environment containing a plasmaspheric plume, Geophys. Res. Lett., 36, L22101, doi:10.1029/2009GL040451.

Chum, J., and O. Santolík (2005), Propagation of whistler-mode chorus to low altitudes: Divergent ray trajectories and ground accessibility, Ann. Geophys., 23, 3727-3738.

Cornilleau-Wehrlin, N., R. Gendrin, F. Lefeuvre, M. Parrot, R. Grard, D. Jones, A. Bahnsen, E. Ungstrup, and W. Gibbons (1978), VLF electromagnetic waves observed onboard GEOS-1, Space Sci. Rev., 22, 371-382.

Dunckel, N., and R. A. Helliwell (1969), Whistler-mode emissions on the OGO 1 satellite, J. Geophys. Res., 74, 6371-6385, doi:10.1029/ JA074i026p06371.

Edgar, B. C. (1976), The upper- and lower-frequency cutoffs of magnetospherically reflected whistlers, J. Geophys. Res., 81, 205-211, doi:10.1029/JA081i001p00205

Gail, W. B., and D. L. Carpenter (1984), Whistler-induced suppression of VLF noise, J. Geophys. Res., 89, 1015-1022, doi:10.1029/ JA089iA02p01015.

Gallagher, D. L., P. D. Craven, and R. H. Comfort (2000), Global core plasma model, J. Geophys. Res., 105, 18,819-18,834, doi:10.1029/ 1999JA000241.

Golden, D. I., M. Spasojevic, and U. S. Inan (2009), Diurnal dependence of $\mathrm{ELF} / \mathrm{VLF}$ hiss and its relation to chorus at $\mathrm{L}=2.4, \mathrm{~J}$. Geophys. Res., 114, A05212, doi:10.1029/2008JA013946.

Goldstein, J., M. Spasojević, P. H. Reiff, B. R. Sandel, W. T. Forrester, D. L. Gallagher, and B. W. Reinisch (2003), Identifying the plasmapause in IMAGE EUV data using IMAGE RPI in situ steep density gradients, J. Geophys. Res., 108(A4), 1147, doi:10.1029/2002JA009475.

Gurnett, D. A., and B. J. O'Brien (1964), High-latitude geophysical studies with satellite Injun 3: 5. Very low frequency electromagnetic radiation, J. Geophys. Res., 69, 65-89.

Haque, N., M. Spasojevic, O. Santolík, and U. S. Inan (2010), Wave normal angles of magnetospheric chorus emissions observed on the Polar spacecraft, J. Geophys. Res., 115, A00F07, doi:10.1029/2009JA014717.

Haselgrove, J. (1955), Ray theory and a new method for ray tracing, in The Physics of the Ionosphere: Report of the Physical Society Conference on the Physics of the Ionosphere Held at the Cavendish Laboratory, Cambridge, September, 1954, p. 355, Physical Soc., London.

Hayakawa, M., Y. Yamanaka, M. Parrot, and F. Lefeuvre (1984), The wave normals of magnetospheric chorus emissions observed on board GEOS 2 J. Geophys. Res., 89, 2811-2821, doi:10.1029/JA089iA05p02811.

Helliwell, R. A. (1965), Whistlers and Related Ionospheric Phenomena, Stanford Univ. Press, Stanford, Calif

Horne, R. B., S. A. Glauert, and R. M. Thorne (2003), Resonant diffusion of radiation belt electrons by whistler-mode chorus, Geophys. Res. Lett. 30(9), 1493, doi:10.1029/2003GL016963.

Horne, R. B., et al. (2005), Wave acceleration of electrons in the Van Allen radiation belts, Nature, 437, 227-230, doi:10.1038/nature03939.

Imhof, W. L., H. D. Voss, J. Mobilia, M. Walt, and U. S. Inan (1989), Characteristics of short-duration electron precipitation bursts and their relationship with VLF wave activity, J. Geophys. Res., 94, 10,07910,093, doi:10.1029/JA094iA08p10079.

Inan, U. S., and T. F. Bell (1977), The plasmapause as a VLF wave guide, J. Geophys. Res., 82, 2819-2827, doi:10.1029/JA082i019p02819.

Kennel, C. (1966), Low-frequency whistler mode, Phys. Fluids, 9 , 2190-2202, doi:10.1063/1.1761588

Kimura, I. (1966), Effects of ions on whistler-mode ray tracing, Radio Sci., 1(3), 269-283.

LeDocq, M. J., D. A. Gurnett, and G. B. Hospodarsky (1998), Chorus source locations from VLF Poynting flux measurements with the Polar spacecraft, Geophys. Res. Lett., 25, 4063-4066, doi:10.1029/ 1998GL900071.

Lehtinen, N. G., and U. S. Inan (2008), Radiation of ELF/VLF waves by harmonically varying currents into a stratified ionosphere with applica- 
tion to radiation by a modulated electrojet, J. Geophys. Res., 113, A06301, doi:10.1029/2007JA012911.

Lehtinen, N. G., and U. S. Inan (2009), Full-wave modeling of transionospheric propagation of VLF waves, Geophys. Res. Lett., 36, L03104, doi:10.1029/2008GL036535.

Lekien, F., and J. Marsden (2005), Tricubic interpolation in three dimensions, Int. J. Numer. Methods Eng., 63, 455-471.

Li, W., et al. (2009), Global distribution of whistler-mode chorus waves observed on the THEMIS spacecraft, Geophys. Res. Lett., 36, L09104, doi:10.1029/2009GL037595.

Lorentzen, K. R., J. B. Blake, U. S. Inan, and J. Bortnik (2001), Observations of relativistic electron microbursts in association with VLF chorus, J. Geophys. Res., 106, 6017-6028, doi:10.1029/2000JA003018.

Lyons, L. R., and R. M. Thorne (1973), Equilibrium structure of radiation belt electrons, J. Geophys. Res., 78, 2142-2149, doi:10.1029/ JA078i013p02142.

Lyons, L. R., R. M. Thorne, and C. F. Kennel (1972), Pitch-angle diffusion of radiation belt electrons within the plasmasphere, J. Geophys. Res., 77, 3455-3474.

Meredith, N. P., R. B. Horne, and R. R. Anderson (2001), Substorm dependence of chorus amplitudes: Implications for the acceleration of electrons to relativistic energies, J. Geophys. Res., 106, 13,165-13,178, doi:10.1029/2000JA900156.

Meredith, N. P., R. B. Horne, D. Summers, R. M. Thorne, R. H. A. Iles, D. Heynderickx, and R. R. Anderson (2002), Evidence for acceleration of outer zone electrons to relativistic energies by whistler mode chorus, Ann. Geophys., 20, 967-979.

Meredith, N. P., R. B. Horne, S. A. Glauert, and R. R. Anderson (2007) Slot region electron loss timescales due to plasmaspheric hiss and lightning-generated whistlers, J. Geophys. Res., 112, A08214, doi:10.1029/ 2007JA012413.

O’Brien, T. P., K. R. Lorentzen, I. R. Mann, N. P. Meredith, J. B. Blake, J. F. Fennell, M. D. Looper, D. K. Milling, and R. R. Anderson (2003), Energization of relativistic electrons in the presence of ULF power and $\mathrm{MeV}$ microbursts: Evidence for dual ULF and VLF acceleration, J. Geophys. Res., 108(A8), 1329, doi:10.1029/2002JA009784.

Parrot, M., O. Santolík, D. Gurnett, J. Pickett, and N. Cornilleau-Wehrlin (2004), Characteristics of magnetospherically reflected chorus waves observed by CLUSTER, Ann. Geophys., 22, 2597-2606.

Roelof, E. C., and A. J. Skinner (2000), Extraction of ion distributions from magnetospheric ENA and EUV images, Space Sci. Rev., 91, 437-459.

Sandel, B. R., et al. (2000), The Extreme Ultraviolet Imager investigation for the IMAGE mission, Space Sci. Rev., 91, 197-242.

Santolík, O. (2008), New results of investigations of whistler-mode chorus emissions, Nonlinear Processes Geophys., 15, 621-630.

Santolík, O., D. A. Gurnett, J. S. Pickett, M. Parrot, and N CornilleauWehrlin (2005), Central position of the source region of storm-time chorus, Planet. Space Sci., 53, 299-305, doi:10.1016/j.pss.2004.09.056.

Santolík, O., J. Chum, M. Parrot, D. A. Gurnett, J. S. Pickett, and N. Cornilleau-Wehrlin (2006), Propagation of whistler mode chorus to low altitudes: Spacecraft observations of structured ELF hiss, J. Geophys. Res., 111, A10208, doi:10.1029/2005JA011462.
Shprits, Y. Y., R. M. Thorne, R. B. Horne, S. A. Glauert, M. Cartwright, C. T. Russell, D. N. Baker, and S. G. Kanekal (2006), Acceleration mechanism responsible for the formation of the new radiation belt during the 2003 Halloween solar storm, Geophys. Res. Lett., 33, L05104, doi:10.1029/2005GL024256.

Smith, A. J., M. P. Freeman, M. G. Wickett, and B. D. Cox (1999), On the relationship between the magnetic and VLF signatures of the substorm expansion phase, J. Geophys. Res., 104, 12,351-12,360, doi:10.1029/ 1998JA900184.

Smith, R. L. (1961), Propagation characteristics of whistlers trapped in field-aligned columns of enhanced ionization, J. Geophys. Res., 66 3699-3707, doi:10.1029/JZ066i011p03699.

Sonwalkar, V. S. (1995), Magnetospheric LF-, VLF-, and ELF-waves, in Handbook of Atmospheric Electrodynamics, vol. 2, edited by H. Volland, pp. 407-460, CRC Press, Boca Raton, Fla.

Sonwalkar, V. S., and J. Harikumar (2000), An explanation of ground observations of auroral hiss: Role of density depletions and meter-scale irregularities, J. Geophys. Res., 105, 18,867-18,884, doi:10.1029/ 1999JA000302

Spasojevic, M., and U. S. Inan (2010), Drivers of chorus in the outer dayside magnetosphere, J. Geophys. Res., 115, A00F09, doi:10.1029/ 2009JA014452.

Thorne, R. M., S. R. Church, W. J. Malloy, and B. T. Tsurutani (1977), The local time variation of ELF emissions during periods of substorm activity, J. Geophys. Res., 82, 1585-1590.

Thorne, R. M., T. P. O'Brien, Y. Y. Shprits, D. Summers, and R. B. Horne (2005), Timescale for $\mathrm{MeV}$ electron microburst loss during geomagnetic storms, J. Geophys. Res., 110, A09202, doi:10.1029/2004JA010882.

Tsurutani, B. T., and E. J. Smith (1974), Postmidnight chorus: A substorm phenomenon, J. Geophys. Res., 79, 118-127, doi:10.1029/ JA079i001p00118.

Tsurutani, B. T., and E. J. Smith (1977), Two types of magnetospheric ELF chorus and their substorm dependences, J. Geophys. Res., 82, 5112-5128.

Tsyganenko, N. A. (1995), Modeling the Earth's magnetospheric magnetic field confined within a realistic magnetopause, J. Geophys. Res., 100, 5599-5612, doi:10.1029/94JA03193.

Tsyganenko, N. A., and D. P. Stern (1996), Modeling the global magnetic field of the large-scale Birkeland current systems, J. Geophys. Res., 101, 27,187-27,198, doi:10.1029/96JA02735.

Walt, M. (1994), Introduction to Geomagnetically Trapped Radiation, Cambridge Univ. Press, Cambridge, U. K.

F. R. Foust, D. I. Golden, N. G. Lehtinen, and M. Spasojevic, STAR Laboratory, Stanford University, 350 Serra Mall, Packard Bldg., Stanford, CA 94305-9515, USA. (dgolden1@stanford.edu)

U. S. Inan, Electrical Engineering Department, Koc University, Sariyer 34450, Istanbul, Turkey. (inan@stanford.edu)

N. P. Meredith, British Antarctic Survey, Natural Environment Research Council, Madingley Road, Cambridge CB3 0ET, UK. (nmer@bas.ac.uk) 